\title{
Single Higgs production in association with a photon at electron-positron colliders in extended Higgs models
}

\author{
Shinya Kanemura, ${ }^{1}$ Kentarou Mawatari, ${ }^{1}$ and Kodai Sakurai ${ }^{2,1}$ \\ ${ }^{1}$ Department of Physics, Osaka University, Toyonaka, Osaka 560-0043, Japan \\ ${ }^{2}$ Department of Physics, University of Toyama, 3190 Gofuku, Toyama 930-8555, Japan
}

(Received 30 August 2018; published 15 February 2019)

\begin{abstract}
We study associated Higgs production with a photon at electron-positron colliders, $e^{+} e^{-} \rightarrow h \gamma$, in various extended Higgs models, such as the inert doublet model, the inert triplet model, and the two Higgs doublet model. The cross section in the standard model (SM) is maximal around $\sqrt{s}=250 \mathrm{GeV}$, and we present how and how much the new physics can enhance or reduce the production rate. We also discuss the correlation with the $h \rightarrow \gamma \gamma$ and $h \rightarrow Z \gamma$ decay rates. We find that, with a sizable coupling to a SM-like Higgs boson, charged scalars can give considerable contributions to both the production and the decay if their masses are around $100 \mathrm{GeV}$. Under the theoretical constraints from vacuum stability and perturbative unitarity as well as the current constraints from the Higgs measurements at the LHC, the production rate can be enhanced from the SM prediction at most by a factor of 2 in the inert doublet model. In the inert triplet model, in addition, we find a particular parameter region where the $h \gamma$ production significantly increases by a factor of about 6 to 8, but the $h \rightarrow \gamma \gamma$ decay still remains as in the SM. In the two Higgs doublet model, possible deviations from the SM prediction are minor in the viable parameter space.
\end{abstract}

DOI: 10.1103/PhysRevD.99.035023

\section{INTRODUCTION}

High-energy $e^{+} e^{-}$colliders as a Higgs factory have been discussed for a long time in order to identify the Higgs sector; i.e., a mechanism of electroweak symmetry breaking and its relation to physics beyond the standard model (SM). Especially after the discovery of the $125 \mathrm{GeV}$ Higgs boson in $p p$ collisions at the LHC, the physics potential for precise measurements of the Higgs couplings at $\sqrt{s}=$ $240-250 \mathrm{GeV}$ has been extensively studied for realization of the International Linear Collider (ILC) [1-3] as well as the lepton collision option of the Future Circular Collider [4] and the Circular Electron Positron Collider [5]. Such collision energies are optimal for studying associated Higgs production with a $Z$ boson, $e^{+} e^{-} \rightarrow h Z$, where the total cross section is maximal around $\sqrt{s}=250 \mathrm{GeV}$ in the $\mathrm{SM}$.

On the other hand, it is less known that with the mass of $m_{h}=125 \mathrm{GeV}$ the cross section for associated Higgs production with a photon, $e^{+} e^{-} \rightarrow h \gamma$, also has a peak around $\sqrt{s}=250 \mathrm{GeV}$ in the SM [6,7]. Since the process is protected by the electromagnetic gauge symmetry and the tree-level contribution is highly suppressed by the electron mass, it is essentially loop induced, and hence

Published by the American Physical Society under the terms of the Creative Commons Attribution 4.0 International license. Further distribution of this work must maintain attribution to the author(s) and the published article's title, journal citation, and DOI. Funded by SCOAP ${ }^{3}$. the cross section is rather small, $\mathcal{O}(0.1) \mathrm{fb}$ at $\sqrt{s}=$ $250 \mathrm{GeV}$. However, because the signal is very clean, i.e., a monochromatic photon in the final state, the above future colliders with the design luminosity may be able to observe the signal. Moreover, new physics can substantially enhance the production rate relative to the SM case; i.e., the process has the potential to explore physics beyond the SM. In this work, we study how and how much new physics can enhance (or reduce) the $h \gamma$ signal at future $e^{+} e^{-}$colliders.

The process was studied in the SM a long time ago [6-8]. The next-to-leading-order QCD corrections were recently estimated in Ref. [9], which shows negligible impact at lower center-of-mass energies $\sqrt{s}<300 \mathrm{GeV}$. On the other hand, new physics effects on the process have not been fully explored yet. There are several studies in the literature in the context of anomalous Higgs-boson couplings or an effective field theory [10-15] as well as concrete new physics models such as supersymmetric models [7,16-18] and the inert doublet model (IDM) [19].

In this article, we study the $e^{+} e^{-} \rightarrow h \gamma$ process in various extended Higgs models, including the IDM studied in Ref. [19], the inert triplet model (ITM), and two Higgs doublet models (THDMs) as distinctive benchmark models for the process. We calculate the loop-induced amplitudes at the leading order (LO) by employing and extending the H-COUP program [20], which evaluates the renormalized gauge-invariant vertex functions for the SM-like Higgs boson in various extended Higgs models. Through a systematic 
study of the different models, we would like to obtain more general information on new physics effects in the signal.

We begin with a brief review of the $e^{+} e^{-} \rightarrow h \gamma$ cross section in the SM in Sec. II. After introducing each extended Higgs model in Sec. III, we present the cross sections in each model and also discuss the correlations with the $h \rightarrow \gamma \gamma$ and $h \rightarrow Z \gamma$ decay rates in Sec. IV. The results are shown not only at $\sqrt{s}=250 \mathrm{GeV}$ but also at $\sqrt{s}=500 \mathrm{GeV}$ in anticipation of the energy extension of the ILC or the Compact Linear Collider [21]. The summary is given in Sec. V. Explicit formulas for the $h \gamma \gamma$ and $h Z \gamma$ vertex functions are listed in the Appendix.

\section{PROCESS OF $e^{+} e^{-} \rightarrow h \gamma$ IN THE SM}

We briefly review the total cross section for $e^{+} e^{-} \rightarrow h \gamma$ in the SM [6-9]. As mentioned in Sec. I, the process is loop induced, and we evaluate it at LO. The details of the calculation will be discussed in Sec. IV.

Figure 1 shows the total cross section $\sigma\left(e^{+} e^{-} \rightarrow h \gamma\right)$ in the SM as a function of the collision energy $\sqrt{s}{ }^{1}$ One can observe an interesting peak-dip structure due to negative interference among different contributions [7,9]. To understand the structure, we decompose all the contributions into two categories in the 't Hooft-Feynman gauge; one is contributions from triangle $h V \gamma(V=\gamma$ or $Z)$ loops, and the other is from box loops, shown in blue and green lines, respectively, in Fig. 1. The $h V \gamma$ contributions are further decomposed into the ones from top quarks and from $W$ bosons, denoted by blue dashed and dotted lines, respectively. We depict the representative Feynman diagrams in Fig. 2. The contribution from fermion loops [diagram (a)], mainly from top quarks, becomes maximum around the $t \bar{t}$ threshold, leading to the dip structure for the total rate. $W$-boson loops in the triangle loop contribution [diagram (b)] are dominant over the top-quark loops, especially $\sqrt{s}<2 m_{t}$. The box contribution includes $W / \nu$ loops [diagram (c)] as well as a $Z / e$ loop. Combining all the contributions at the amplitude level, the total cross section eventually has the first peak at $\sqrt{s} \sim 250 \mathrm{GeV}$ and the second one at $\sqrt{s} \sim 500 \mathrm{GeV}$. As we see, the production rate is very sensitive to the magnitudes of each amplitude as well as the relative phases among them. Therefore, slight modifications of the SM interactions as well as loop contributions from new particles can substantially enhance (or reduce) the production rate. We note that the relative magnitudes between the triangle and box contributions are rather different at different energies, and hence new physics can affect the total rates differently at different collision energies, e.g., between $\sqrt{s}=250$ and $500 \mathrm{GeV}$, as seen below.

\footnotetext{
${ }^{1}$ The cross section is shown with unpolarized beams. With fully polarized left-handed electrons and right-handed positrons, the cross section is approximately four times larger than in the unpolarized case [7].
}

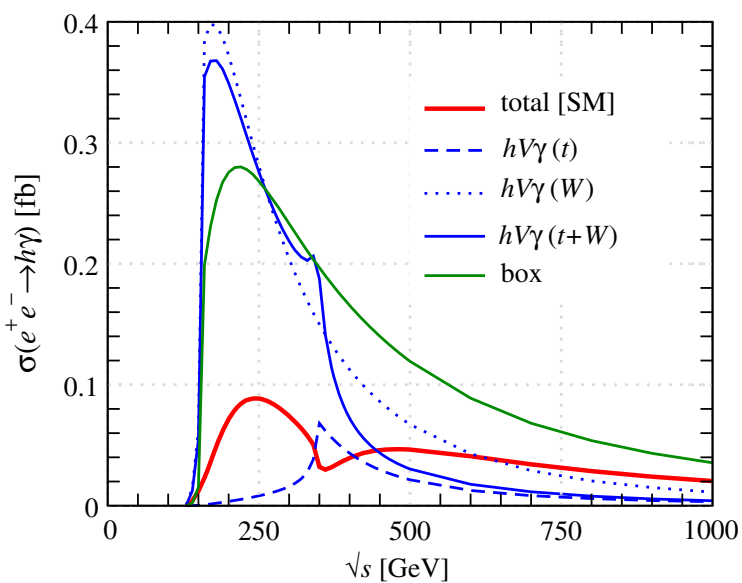

FIG. 1. Total cross section for $e^{+} e^{-} \rightarrow h \gamma$ in the SM as a function of the collision energy. The total rate (red solid) is decomposed into two contributions; triangle loops (blue solid) and box loops (green solid). The contribution from the triangle loops is further decomposed into the ones from top quarks (blue dashed) and from $W$ bosons (blue dotted).

Before turning to the next section, we discuss how new physics can affect the process. In extended Higgs models, the SM-like Higgs interactions can be modified due to the mixing with other neutral scalars. The effect is described as the tree-level scaling factors

$$
\kappa_{X}=\frac{g_{h X X}^{\mathrm{NP}}}{g_{h X X}^{\mathrm{SM}}},
$$

the ratio of the Higgs coupling in a new physics model to the one in the SM, denoted as red blobs in diagrams (a), (b), and (c) in Fig. 2. In addition, the process can be modified by additional triangle loops of charged scalars; see diagram (d). In the following, we study such effects in each extended Higgs model.

\section{EXTENDED HIGGS MODELS}

As discussed above, in extended Higgs models, the $e^{+} e^{-} \rightarrow h \gamma$ process can be altered through 1) the modified couplings of the SM-like Higgs boson and/or 2) charged scalar loops. To study such new physics effects on the process, we consider three distinctive models: the IDM, ITM with the hypercharge $Y=1$, and the THDM. At the tree level, while in the first two models the SM-like Higgs couplings to SM fermions and weak bosons are the same as in the SM, in the THDM, those are modified by the mixing of the scalar fields. While all the three models include singly charged scalars, the ITM includes doubly charged scalars as well. We briefly describe the three models in order. We give the Higgs potential and define the relevant parameters for each model. We also describe theoretical and experimental constraints on the parameters. The experimental constraint that is strongly relevant to the $e^{+} e^{-} \rightarrow h \gamma$ 


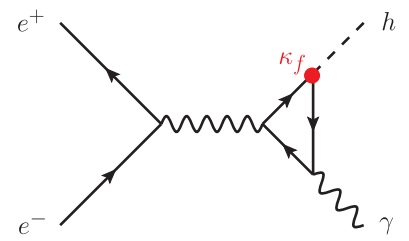

(a)

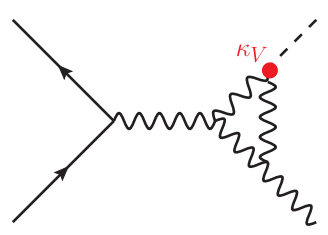

(b)

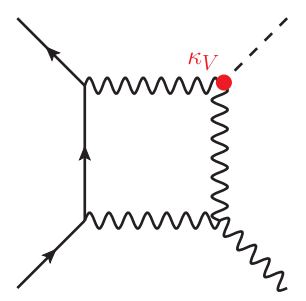

(c)

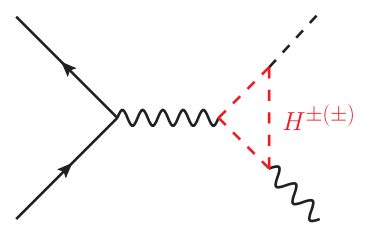

(d)

FIG. 2. Representative Feynman diagrams for $e^{+} e^{-} \rightarrow h \gamma$.

process comes from the $h \rightarrow \gamma \gamma$ signal at the LHC, which will be discussed exceptionally in the next section.

\section{A. Inert doublet model}

In the IDM [22,23], an isospin doublet field $\Phi_{2}$ with hypercharge $Y=1 / 2$ is added to the SM Higgs doublet field $\Phi_{1}$. The model imposes an exact $Z_{2}$ symmetry under which $\Phi_{2}$ is odd while all the other fields are even. The general Higgs potential under the $Z_{2}$ symmetry is given by

$$
\begin{aligned}
V\left(\Phi_{1}, \Phi_{2}\right)= & \mu_{1}^{2}\left|\Phi_{1}\right|^{2}+\mu_{2}^{2}\left|\Phi_{2}\right|^{2}+\frac{1}{2} \lambda_{1}\left|\Phi_{1}\right|^{4}+\frac{1}{2} \lambda_{2}\left|\Phi_{2}\right|^{4} \\
& +\lambda_{3}\left|\Phi_{1}\right|^{2}\left|\Phi_{2}\right|^{2}+\lambda_{4}\left|\Phi_{1}^{\dagger} \Phi_{2}\right|^{2} \\
& +\frac{1}{2} \lambda_{5}\left\{\left(\Phi_{1}^{\dagger} \Phi_{2}\right)^{2}+\text { H.c. }\right\}
\end{aligned}
$$

where all the parameters can be taken to be real. Because of the $Z_{2}$ symmetry, only $\Phi_{1}$ can have a nonzero vacuum expectation value (VEV) $v$. The two doublet fields $\Phi_{1,2}$ are parametrized as

$\Phi_{1}=\left(\begin{array}{c}G^{+} \\ \frac{1}{\sqrt{2}}\left(v+h+i G^{0}\right)\end{array}\right), \quad \Phi_{2}=\left(\begin{array}{c}H^{+} \\ \frac{1}{\sqrt{2}}(H+i A)\end{array}\right)$,

where $G^{ \pm, 0}$ are Nambu-Goldstone bosons to be absorbed by weak bosons. $h$ is the SM-like Higgs boson with the mass of $125 \mathrm{GeV}$, while $H, A$, and $H^{ \pm}$are $Z_{2}$-odd inert scalar bosons. After imposing the stationary condition, masses of the scalar bosons are written in terms of the parameters in the potential as

$$
\begin{array}{ll}
m_{h}^{2}=-2 \mu_{1}^{2}=v^{2} \lambda_{1}, & m_{H^{ \pm}}^{2}=\mu_{2}^{2}+\frac{1}{2} v^{2} \lambda_{3}, \\
m_{H}^{2}=\mu_{2}^{2}+\frac{1}{2} v^{2} \lambda_{345}^{+}, & m_{A}^{2}=\mu_{2}^{2}+\frac{1}{2} v^{2} \lambda_{345}^{-},
\end{array}
$$

with $\lambda_{345}^{ \pm} \equiv \lambda_{3}+\lambda_{4} \pm \lambda_{5}$. We choose the following five parameters in the $\mathrm{IDM}^{2}$ :

\footnotetext{
${ }^{2}$ Instead of $\lambda_{3}$, the H-COUP program uses $\mu_{2}^{2}$ as an input parameter.
}

$$
m_{H}, \quad m_{A}, \quad m_{H^{+}}, \quad \lambda_{2}, \quad \lambda_{3} .
$$

Because of the exact $Z_{2}$ symmetry, none of the inert scalars has direct couplings to SM fermions. The couplings of the SM-like Higgs boson to SM fermions and weak bosons are exactly the same as in the SM, i.e.,

$$
\kappa_{f}=\kappa_{V}=1
$$

in Eq. (1). ${ }^{3}$ While the couplings of the charged scalars to gauge bosons are the SM gauge couplings, the coupling to the SM-like Higgs boson is given by

$$
g_{h H^{+} H^{-}}=-\frac{2}{v}\left(m_{H^{+}}^{2}-\mu_{2}^{2}\right)=-v \lambda_{3} .
$$

We note that, if $\lambda_{3}=0$ ( or $m_{H^{+}}^{2}=\mu_{2}^{2}$ ), the predictions for $e^{+} e^{-} \rightarrow h \gamma$ in the IDM and in the SM are identical at the LO.

The following theoretical and experimental constraints on the above parameters are taken into account $[26,27]$. Vacuum stability [22] and the existence of the inert vacuum [28] require

$\lambda_{1,2}>0, \quad \sqrt{\lambda_{1} \lambda_{2}}+\lambda_{3}+\operatorname{MIN}\left[0, \lambda_{4}-\lambda_{5}, \lambda_{4}+\lambda_{5}\right]>0$

and

$$
m_{h, H, A, H^{+}}^{2}>0, \quad \frac{\mu_{1}^{2}}{\sqrt{\lambda_{1}}}<\frac{\mu_{2}^{2}}{\sqrt{\lambda_{2}}},
$$

respectively. We evaluate constraints from perturbative unitarity, adopting the formulas given in Refs. [29,30]. ${ }^{4}$

LEP-I precision measurements exclude the possibility that weak bosons decay into a pair of inert scalars; i.e., $m_{H^{+}}+m_{H, A}>m_{W}, 2 m_{H^{+}}>m_{Z}$, and $m_{H}+m_{A}>m_{Z}$. Moreover, the electroweak precision data, especially the $T$ parameter, imply that a mass difference between charged scalars $\left(H^{ \pm}\right)$and a neutral inert scalar $(H$ or $A)$ must not be too large $[23,34]$. We note that direct searches for additional scalars in collider experiments have been often done in the

\footnotetext{
${ }^{3}$ Note that those couplings deviate from the SM prediction at higher orders $[24,25]$.

${ }^{4}$ Notice that if we use criteria other than the tree-level unitarity, such as the unitarity bounds at one-loop level [31] or the triviality bounds $[23,32,33]$, the parameter space can be more restricted.
} 

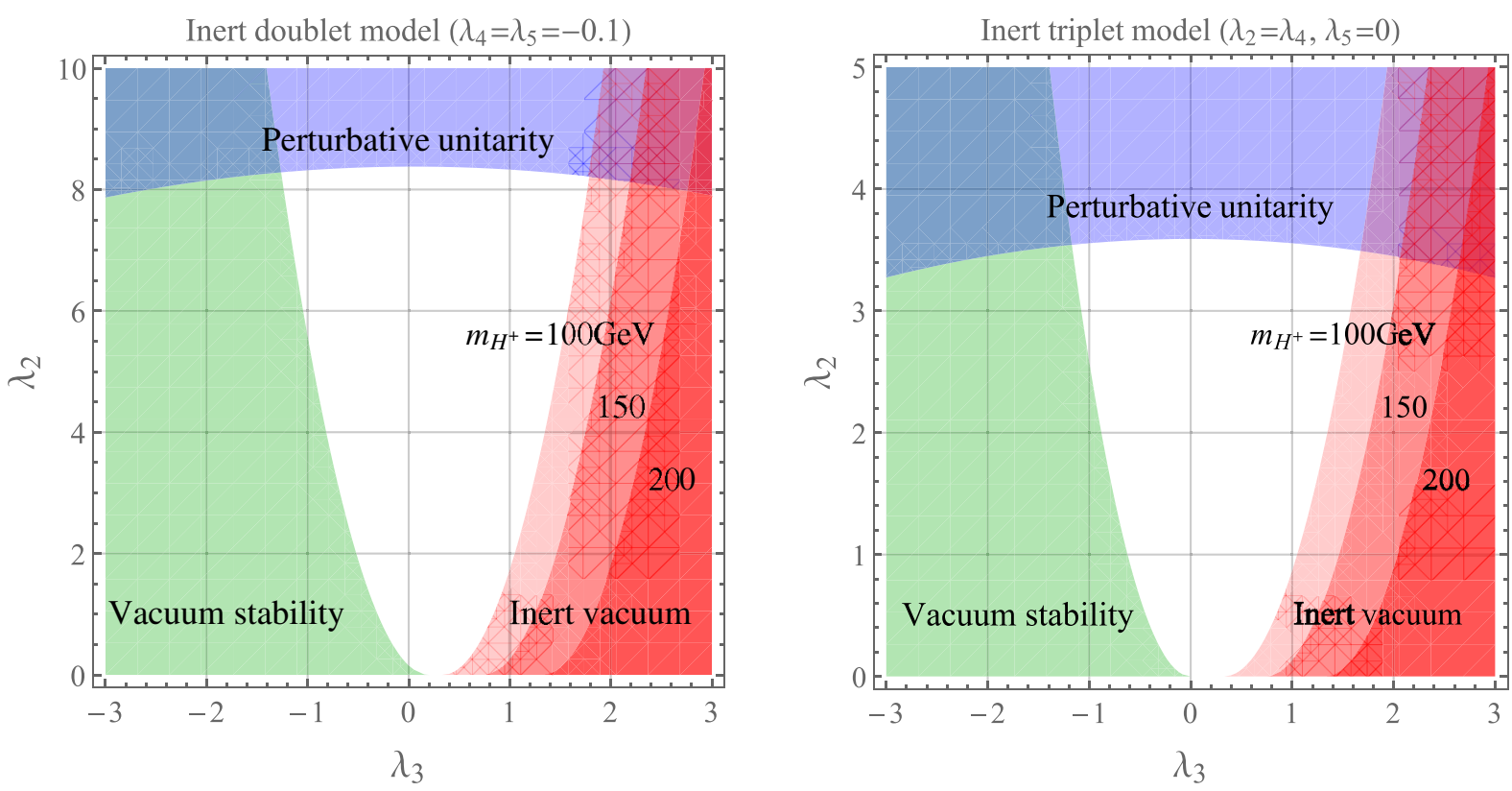

FIG. 3. Theoretical constraints on the $\lambda_{3}-\lambda_{2}$ plane in the IDM (left) and in the ITM (right).

context of THDMs or supersymmetric models, in which the scalar particles couple to SM fermions. Therefore, careful reinterpretations are necessary to constrain the inert models. LEP-II chargino search results are naively interpreted as $m_{H^{+}}>70-90 \mathrm{GeV}$ [35], while neutralino search results exclude the region where simultaneously $m_{H}<80 \mathrm{GeV}$, $m_{A}<100 \mathrm{GeV}$, and $m_{A}-m_{H}>8 \mathrm{GeV}$, where $m_{H}<m_{A}$ is assumed [36,37]. The constraints from the $8 \mathrm{TeV}$ LHC data have been also studied recently [34,38].

In our study, the value of the coupling $g_{h H^{+} H^{-}}$in Eq. (7), i.e., $\lambda_{3}$, is one of the relevant parameters. Figure 3 (left) shows allowed regions of $\lambda_{3}$ as a function of $\lambda_{2}$ in the IDM after imposing the above theoretical constraints. Here, we take $\lambda_{4}=\lambda_{5}=-0.1$ as a benchmark to give a mass spectrum as $m_{H}<m_{H^{+}}=m_{A}{ }^{5} \lambda_{3}$ is bounded below by the vacuum stability (green region) and above by the existence of the inert vacuum (red). The constraint from the inert vacuum depends on the charged scalar mass. Those constrains depend on $\lambda_{2}$, which is bounded above by perturbative unitarity (blue).

We note that the IDM has been often studied in the context of dark matter $[23,39,40]$ since the lightest neutral inert scalar can be stable due to the discrete $Z_{2}$ symmetry. Although we do not consider such a property in this work, dark matter constraints, such as from relic density and direct detection, can also limit the parameter space; see, e.g., Ref. [34] for details. ${ }^{6}$

\footnotetext{
${ }^{5}$ See Ref. [26] for the allowed region in detail.

${ }^{6}$ Especially, the recent result of the XENON-1T dark matter direct detection [41] provides a strong limit on the mass of the dark matter candidate, i.e., the lightest inert scalar, as well as $\lambda_{345}$ [42]. We note that, as mentioned below in footnote 9, we can still find interesting parameter regions which are compatible with dark matter constraints.
}

\section{B. Inert triplet model}

Instead of a doublet field as in the IDM, we introduce an isospin triplet field $\Delta$ with hypercharge $Y=1$ and impose an exact $Z_{2}$ symmetry under which $\Delta$ is odd while all the other SM fields are even $[43,44]$. The Higgs potential is given by ${ }^{7}$

$$
\begin{aligned}
V(\Phi, \Delta)= & \mu_{1}^{2}|\Phi|^{2}+\mu_{2}^{2} \operatorname{Tr}\left[\Delta^{\dagger} \Delta\right]+\frac{1}{2} \lambda_{1}|\Phi|^{4} \\
& +\frac{1}{2} \lambda_{2}\left(\operatorname{Tr}\left[\Delta^{\dagger} \Delta\right]\right)^{2}+\lambda_{3}|\Phi|^{2} \operatorname{Tr}\left[\Delta^{\dagger} \Delta\right] \\
& +\frac{1}{2} \lambda_{4} \operatorname{Tr}\left[\left(\Delta^{\dagger} \Delta\right)^{2}\right]+\lambda_{5} \Phi^{\dagger} \Delta \Delta^{\dagger} \Phi,
\end{aligned}
$$

where all the parameters are real and the fields are parametrized by

$$
\begin{aligned}
& \Phi=\left(\begin{array}{c}
G^{+} \\
\frac{1}{\sqrt{2}}\left(v+h+i G^{0}\right)
\end{array}\right), \\
& \Delta=\left(\begin{array}{cc}
\frac{1}{\sqrt{2}} H^{+} & H^{++} \\
\frac{1}{\sqrt{2}}(H+i A) & -\frac{1}{\sqrt{2}} H^{+}
\end{array}\right) .
\end{aligned}
$$

In addition to singly charged scalars, the model contains doubly charged scalars. The mass spectrum for the inert scalars is given by

$$
\begin{aligned}
m_{H^{ \pm \pm}}^{2} & =\mu_{2}^{2}+\frac{1}{2} v^{2} \lambda_{3}, \quad m_{H, A}^{2}=\mu_{2}^{2}+\frac{1}{2} v^{2}\left(\lambda_{3}+\lambda_{5}\right), \\
m_{H^{ \pm}}^{2} & =\frac{1}{2}\left(m_{H^{ \pm \pm}}^{2}+m_{H}^{2}\right)
\end{aligned}
$$

\footnotetext{
${ }^{7}$ For comparison with the IDM, we adopt the convention in Ref. [43] for the definition of the potential, which differs from the usual convention in which $\lambda_{3}$ and $\lambda_{4}$ are exchanged.
} 
while the mass of the SM-like Higgs boson is $m_{h}^{2}=$ $-2 \mu_{1}^{2}=v^{2} \lambda_{1}$. We choose the following five parameters in the ITM:

$$
m_{H}, \quad m_{H^{++}}, \quad \lambda_{2}, \quad \lambda_{3}, \quad \lambda_{4} .
$$

Similar to the IDM, the inert scalars do not couple to SM fermions, and the SM-like Higgs boson interacts with SM fermions and weak bosons as in the SM, i.e.,

$$
\kappa_{f}=\kappa_{V}=1
$$

The couplings of the charged scalars to the SM-like Higgs boson are given by

$$
\begin{gathered}
g_{h H^{++} H^{--}}=-\frac{2}{v}\left(m_{H^{++}}^{2}-\mu_{2}^{2}\right)=-v \lambda_{3}, \\
g_{h H^{+} H^{-}}=-\frac{2}{v}\left\{\frac{1}{2}\left(m_{H^{++}}^{2}+m_{H}^{2}\right)-\mu_{2}^{2}\right\}=-v\left(\lambda_{3}+\frac{1}{2} \lambda_{5}\right) .
\end{gathered}
$$

We note that if $\lambda_{5}=0$ the doubly and singly charged scalars have the same mass and the same coupling to the SM-like Higgs boson. If $\lambda_{3}=\lambda_{5}=0$, i.e., $m_{H^{++}}^{2}=m_{H^{+}}^{2}=$ $\mu_{2}^{2}$, the predictions for $e^{+} e^{-} \rightarrow h \gamma$ in the ITM and in the SM are identical at LO.

The above parameters should satisfy the following theoretical and experimental constraints. With the squared masses in Eq. (12) positive, vacuum stability [45] and the existence of the inert vacuum require

$$
\begin{aligned}
& \lambda_{1}>0, \quad \lambda_{2}+\operatorname{MIN}\left[\lambda_{4}, \lambda_{4} / 2\right]>0, \\
& \lambda_{3}+\operatorname{MIN}\left[0, \lambda_{5}\right]+\operatorname{MIN}\left[\sqrt{\lambda_{1}\left(\lambda_{2}+\lambda_{4}\right)},\right. \\
& \left.\sqrt{\lambda_{1}\left(\lambda_{2}+\lambda_{4} / 2\right)}\right]>0,
\end{aligned}
$$

and

$$
\frac{\mu_{1}^{2}}{\sqrt{\lambda_{1}}}<\frac{\mu_{2}^{2}}{\sqrt{\lambda_{2}+\lambda_{4}}}
$$

respectively. We evaluate constraints from perturbative unitarity adopting the formulas given in Refs. [45-47].

The constraints on singly charged scalars from LEP measurements are similar to the ones in the IDM described above [48]. Doubly charged scalars have been searched at the LEP [49-51], Tevatron [52,53], and LHC [54,55] and were excluded up to $m_{H^{++}} \sim 400-550 \mathrm{GeV}$. However, those analyses assume a $100 \%$ branching ratio for the $H^{ \pm \pm}$decay to charged leptons, and hence those limit cannot be applied for the ITM. Constraints on the ITM at the LHC were studied in Ref. [56] but have not been fully explored yet. We note that upper limits on the cross section times the branching ratio for doubly charged Higgs boson produced in vector-boson fusion, $\sigma\left(p p \rightarrow H^{ \pm \pm} j j\right) \times B\left(H^{ \pm \pm} \rightarrow W^{ \pm} W^{ \pm}\right)$, was reported recently in Ref. [57].

Similar to the IDM, one of the relevant parameters is $\lambda_{3}$. In Fig. 3 (right), we show allowed regions of $\lambda_{3}$ as a function of $\lambda_{2}$ in the ITM after imposing the theoretical constraints from vacuum stability (green region), the existence of the inert vacuum (red), and perturbative unitarity (blue), where we take $\lambda_{2}=\lambda_{4}$ and $\lambda_{5}=0$ for simplicity. ${ }^{8}$ The allowed region of $\lambda_{3}$ in the ITM is slightly smaller than that in the IDM.

We note that the ITM with hypercharge $Y=0$ does not contain doubly charged scalars, and hence the situation is very similar to that in the IDM in our study. Similar to the IDM, the ITM has been studied in the context of dark matter $[43,44,56]$. For the hypercharge $Y=1$ case, however, most of the parameter space is already excluded by the direct detection experiments due to the $Z$-mediated treelevel scattering $[43,44]{ }^{9}$ Here, we consider the ITM with $Y=1$ as a benchmark model just to describe new physics effects on the $e^{+} e^{-} \rightarrow h \gamma$ processes.

\section{Two Higgs doublet models}

The THDM is similar to the IDM, but instead of imposing an exact $Z_{2}$ symmetry, the model allows softly broken $Z_{2}$ symmetric terms, which still avoids flavorchanging neutral currents at tree level [62]. The Higgs potential in the THDM is given by

$$
\begin{aligned}
V\left(\Phi_{1}, \Phi_{2}\right)= & m_{1}^{2}\left|\Phi_{1}\right|^{2}+m_{2}^{2}\left|\Phi_{2}\right|^{2}-\left(m_{3}^{2} \Phi_{1}^{\dagger} \Phi_{2}+\text { H.c. }\right) \\
& +\frac{1}{2} \lambda_{1}\left|\Phi_{1}\right|^{4}+\frac{1}{2} \lambda_{2}\left|\Phi_{2}\right|^{4}+\lambda_{3}\left|\Phi_{1}\right|^{2}\left|\Phi_{2}\right|^{2} \\
& +\lambda_{4}\left|\Phi_{1}^{\dagger} \Phi_{2}\right|^{2}+\frac{1}{2}\left\{\lambda_{5}\left(\Phi_{1}^{\dagger} \Phi_{2}\right)^{2}+\text { H.c. }\right\},
\end{aligned}
$$

where $m_{3}^{2}$ and $\lambda_{5}$ can be real by assuming the $C P$ conservation. The two doublet fields $\Phi_{1,2}$ are parametrized as

$$
\Phi_{i}=\left(\begin{array}{c}
w_{i}^{+} \\
\frac{1}{\sqrt{2}}\left(v_{i}+h_{i}+i z_{i}\right)
\end{array}\right) \quad \text { with } \quad i=1,2,
$$

where $v_{1}$ and $v_{2}$ are the VEVs of the Higgs doublet fields with $v=\left(v_{1}^{2}+v_{2}^{2}\right)^{1 / 2}$. The mass eigenstates of the Higgs fields are defined as

$$
\begin{aligned}
\left(\begin{array}{l}
h_{1} \\
h_{2}
\end{array}\right) & =R(\alpha)\left(\begin{array}{l}
H \\
h
\end{array}\right), \quad\left(\begin{array}{l}
z_{1} \\
z_{2}
\end{array}\right)=R(\beta)\left(\begin{array}{c}
G^{0} \\
A
\end{array}\right), \\
\left(\begin{array}{l}
w_{1}^{ \pm} \\
w_{2}^{ \pm}
\end{array}\right) & =R(\beta)\left(\begin{array}{l}
G^{ \pm} \\
H^{ \pm}
\end{array}\right),
\end{aligned}
$$

\footnotetext{
${ }^{8}$ See Ref. [58] for the $\lambda_{5} \neq 0$ case.

${ }^{9}$ By adding additional particle contents in the ITM, we can avoid such dark matter constraints; see, e.g., Refs. [59-61].
} 
where $R(\theta)$ is a rotation matrix with a mixing parameter $\theta$ and $\tan \beta=v_{2} / v_{1}$. After imposing two stationary conditions for $h_{1,2}$, masses of the physical Higgs bosons and the mixing angle $\alpha$ are expressed by

$$
\begin{aligned}
m_{H^{ \pm}}^{2} & =M^{2}-\frac{1}{2} v^{2}\left(\lambda_{4}+\lambda_{5}\right), \quad m_{A}^{2}=M^{2}-v^{2} \lambda_{5} \\
m_{H}^{2} & =M_{11}^{2} c_{\beta-\alpha}^{2}+M_{22}^{2} s_{\beta-\alpha}^{2}-M_{12}^{2} s_{2(\beta-\alpha)}, \\
m_{h}^{2} & =M_{11}^{2} s_{\beta-\alpha}^{2}+M_{22}^{2} c_{\beta-\alpha}^{2}+M_{12}^{2} s_{2(\beta-\alpha)} \\
\tan 2(\beta-\alpha) & =-\frac{2 M_{12}^{2}}{M_{11}^{2}-M_{22}^{2}},
\end{aligned}
$$

where $s_{\theta}$ and $c_{\theta}$ represent $\sin \theta$ and $\cos \theta, M^{2} \equiv m_{3}^{2} / s_{\beta} c_{\beta}$ describes the soft breaking scale of the $Z_{2}$ symmetry, and $M_{i j}^{2}$ are the mass matrix elements for the $C P$-even scalar states in the basis of $\left(h_{1}, h_{2}\right) R(\beta)$ :

$$
\begin{aligned}
& M_{11}^{2}=v^{2}\left(\lambda_{1} c_{\beta}^{4}+\lambda_{2} s_{\beta}^{4}+2 \lambda_{345}^{+} s_{\beta}^{2} c_{\beta}^{2}\right), \\
& M_{22}^{2}=M^{2}+\frac{1}{4} v^{2} s_{2 \beta}^{2}\left(\lambda_{1}+\lambda_{2}-2 \lambda_{345}^{+}\right), \\
& M_{12}^{2}=\frac{1}{2} v^{2} s_{2 \beta}\left(-\lambda_{1} c_{\beta}^{2}+\lambda_{2} s_{\beta}^{2}+\lambda_{345}^{+} c_{2 \beta}\right) .
\end{aligned}
$$

We choose the following free parameters in the THDM:

$$
m_{H}, \quad m_{A}, \quad m_{H^{+}}, \quad M^{2}, \quad \tan \beta, \quad c_{\beta-\alpha} .
$$

Unlike the inert models, additional Higgs bosons in the THDM couple with SM fermions through the Yukawa interaction. We can define four types of interactions under the softly broken $Z_{2}$ symmetry: types I, II, X, and Y, depending on the $Z_{2}$ charge assignment for the righthanded fermions [63-68]. In all four types of THDMs, the up-type Yukawa interaction is modified in the same manner. In the type-II and type-Y THDMs, on the other hand, the down-type Yukawa can be enhanced by $\tan \beta$.

The tree-level couplings of the SM-like Higgs boson to SM fermions and weak bosons are modified via the mixing with the other neutral Higgs boson. The scaling factors $\kappa_{f}$ and $\kappa_{V}$ are

$$
\kappa_{f}=s_{\beta-\alpha}+\zeta_{f} c_{\beta-\alpha}, \quad \kappa_{V}=s_{\beta-\alpha},
$$

where $\zeta_{f}=\cot \beta$ for the up-type Yukawa coupling. The $h-H^{+}-H^{-}$coupling is given by

$$
\begin{aligned}
g_{h H^{+} H^{-}}= & -\frac{2}{v}\left\{\left(m_{H^{+}}^{2}-M^{2}+\frac{1}{2} m_{h}^{2}\right) s_{\beta-\alpha}\right. \\
& \left.-\left(M^{2}-m_{h}^{2}\right) \cot 2 \beta c_{\beta-\alpha}\right\} \equiv-v \lambda_{3}^{\prime} .
\end{aligned}
$$

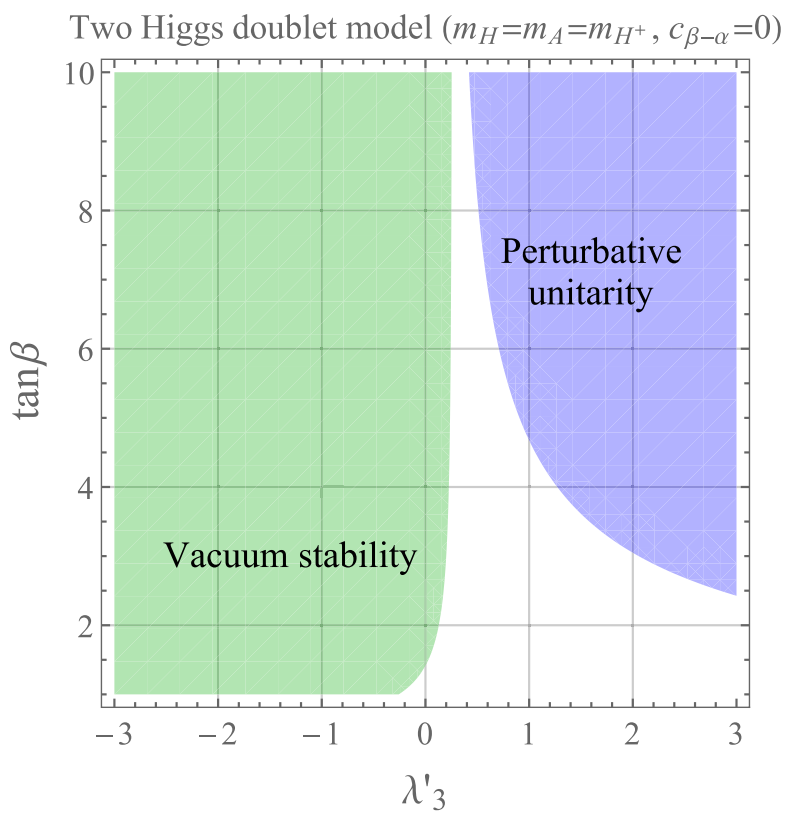

FIG. 4. Theoretical constraints on the $\lambda_{3}^{\prime}-\tan \beta$ plane in the THDM.

For later comparison, $\lambda_{3}^{\prime}$ is defined in the same manner as in Eq. (7) in the IDM.

Regions of the parameter space can be constrained by imposing the bounds from vacuum stability $[22,32,69]$, perturbative unitarity $[29,30,70,71]$, and the $S$ and $T$ parameters [72-76]. Figure 4 shows allowed regions of $\lambda_{3}^{\prime}$ as a function of $\tan \beta$ in the THDM after imposing the above theoretical constraints, where we take $c_{\beta-\alpha}=0$ and $m_{H}=m_{A}=m_{H^{+}}$for an illustration.

Because of the Yukawa interactions of additional Higgs bosons, the parameter space is additionally constrained by the LHC experiments, which are summarized in Ref. [77]. The mass below $160 \mathrm{GeV}$ is excluded from $t \rightarrow H^{ \pm} b$, $H^{ \pm} \rightarrow \tau \nu$ searches for $\tan \beta<4,8,10$ in the type-Y, type-I, and type-X models, respectively. In the type-II model, on the other hand, the same mass region is excluded irrespective of $\tan \beta$. Moreover, flavor experiments such as $B$ meson decays also give an important constraint particularly on the mass of the charged Higgs bosons and $\tan \beta$ [78]. In the type-II (or type-Y) model, $m_{H^{+}}>580 \mathrm{GeV}$ independently of $\tan \beta$. In the type-I (or type-X) model, $m_{H^{+}}>$ $445 \mathrm{GeV}$ for $\tan \beta=1$, while there is no constraint for $\tan \beta>2$. As we see later, for $e^{+} e^{-} \rightarrow h \gamma$, only light charged scalars are relevant, so we only consider the type-I (or equivalently type-X) THDM below.

\section{NUMERICAL RESULTS}

In this section, we describe the formalism of our calculations and show the numerical results at $\sqrt{s}=$ $250 \mathrm{GeV}$ as well as at $\sqrt{s}=500 \mathrm{GeV}$. 


\section{A. Loop calculations by using the H-COUP program}

We consider the process

$$
e^{-}(k, \sigma / 2)+e^{+}(\bar{k}, \bar{\sigma} / 2) \rightarrow \gamma(p, \lambda)+h\left(p_{h}\right),
$$

where the 4-momenta $\left(k, \bar{k}, p, p_{h}\right)$ and helicities $(\sigma, \bar{\sigma}, \lambda)$ are defined in the c.m. frame of the collision. The amplitudes are given by

$$
\mathcal{M}_{\sigma \bar{\sigma}}^{\lambda}=\bar{v}(\bar{k}, \bar{\sigma}) \Gamma^{\nu} u(k, \sigma) \epsilon_{\nu}^{*}(p, \lambda),
$$

where the vertex function $\Gamma^{\nu}$ can be decomposed in terms of the form factors $C_{i}^{\sigma}$ as [7]

$$
\begin{aligned}
\Gamma^{\nu}= & \gamma_{\mu}\left\{C_{1}^{\sigma}\left(g^{\mu \nu} p \cdot k-p^{\mu} k^{\nu}\right)+C_{2}^{\sigma}\left(g^{\mu \nu} p \cdot \bar{k}-p^{\mu} \bar{k}^{\nu}\right)\right. \\
& \left.+C_{3}^{\sigma} g^{\mu \nu}\right\} .
\end{aligned}
$$

$C_{i}^{\sigma}$ sum all the one-loop contributions as

$$
C_{i}^{\sigma}=C_{i}^{h e e, \sigma}+C_{i}^{h V \gamma, \sigma}+C_{i}^{\mathrm{box}, \sigma},
$$

where $C_{i}^{h e e, \sigma}$ denotes the hee vertex contributions, $C_{i}^{h V \gamma, \sigma}$ $(V=\gamma, Z)$ are the triangle vertex contributions [diagrams (a), (b), and (d) in Fig. 2], and $C_{i}^{\text {box, } \sigma}$ is the box contributions [diagram (c)]. Note that, due to the gauge invariance, $C_{3}^{\sigma}$ is zero after summing all the contributions. The differential cross section is [9]

$\frac{d \sigma}{d \cos \theta}=\frac{s-m_{h}^{2}}{64 \pi s}\left\{t^{2}\left(\left|C_{1}^{+}\right|^{2}+\left|C_{1}^{-}\right|^{2}\right)+u^{2}\left(\left|C_{2}^{+}\right|^{2}+\left|C_{2}^{-}\right|^{2}\right)\right\}$,

where $\theta$ is the scattering angle between the electron and the photon in the c.m. frame and $t, u=-\left(s-m_{h}^{2}\right)$ $(1 \mp \cos \theta) / 2$. In the following, we briefly describe how we evaluate the form factors $C_{i}^{\sigma}$ for each loop diagram by using the H-COUP program [20].

$\mathrm{H}$-COUP is a numerical code to evaluate the renormalized gauge-invariant vertex functions for the SM-like Higgs boson at one-loop level in various extended Higgs models [20]. So far, the Higgs singlet model, four types of the THDMs, and the IDM are included, based on Refs. [25,7984]. Recently, the program was extended to include box contributions in order to evaluate the three-body decay $h \rightarrow Z f \bar{f}$ at the one-loop level [85]. Since the $h \rightarrow Z f \bar{f}$ decay is related to the $e^{+} e^{-} \rightarrow h \gamma$ process by crossing symmetry and by replacing the $Z$ boson by the photon, we utilize the program with slight extensions to evaluate the form factors $C_{i}^{\sigma}$ both in the SM and in extended Higgs models. For the ITM, we newly implemented the relevant pieces, based on the IDM. We validated our numerical calculations by comparing with the earlier works in the SM [7,9] as well as in the IDM [19], while the results in the
ITM and the THDMs are reported for the first time in this article.

In the $m_{e}=0$ limit, the one-loop hee vertex in extended Higgs models deviates from the SM prediction only by the mixing effects as the $\kappa_{V}$ scaling factor due to weak-boson loops. However, the hee vertex diagrams are nonzero only for $C_{3}^{\sigma}$, i.e., do not contribute to the physical observable. To check the gauge invariance, we evaluate $C_{3}^{\text {hee, } \sigma}$ by using H-COUP. Similarly, the box contributions in extended Higgs models are modified only by the $\kappa_{V}$ factor. See Ref. [7] for the explicit formulas in the SM.

The most important contribution in extended Higgs models comes from the triangle loop diagrams, especially charged scalar loops. The renormalized $h V \gamma$ vertices are decomposed by the following two form factors in H-COUP,

$$
\Gamma_{h V \gamma}^{\mu \nu}\left(p^{2}, q^{2}, p_{h}^{2}\right)=g^{\mu \nu} \Gamma_{h V \gamma}^{1}-p^{\mu} q^{\nu} \Gamma_{h V \gamma}^{2},
$$

with $q=k+\bar{k}$ being the momentum of the $s$-channel offshell photon or $Z$ boson. As shown in Fig. 2, $\Gamma_{h V \gamma}^{\mu \nu}$ includes top and $W$ loops with the scaling factors $\kappa_{f}$ and $\kappa_{V}$ as well as charged scalar loops in extended Higgs models. $C_{1,2,3}^{h V \gamma, \sigma}$ can be evaluated in terms of $\Gamma_{h V \gamma}^{1,2}$ as

$$
\begin{aligned}
& C_{1}^{h V \gamma, \sigma}=C_{2}^{h V \gamma, \sigma}=-\frac{g_{V e e}^{\sigma}}{s-m_{V}^{2}} \Gamma_{h V \gamma}^{2}, \\
& C_{3}^{h V \gamma, \sigma}=-\frac{g_{V e e}^{\sigma}}{s-m_{V}^{2}}\left(\Gamma_{h V \gamma}^{1}-p \cdot q \Gamma_{h V \gamma}^{2}\right),
\end{aligned}
$$

where $g_{V e e}^{\sigma}$ are the SM $\gamma e e$ and Zee couplings. One can find the explicit expressions for $\Gamma_{h \gamma \gamma}^{1,2}$ and $\Gamma_{h Z \gamma}^{1,2}$ in the Appendix. For the validity of our program, we numerically check the gauge invariance; i.e., $\Gamma_{h V \gamma}^{1}=p \cdot q \Gamma_{h V \gamma}^{2}$ for top and charged scalar loops independently, and $C_{3}^{\sigma}=0$ for weak-boson loops after summing all the contributions. When $q^{2}=0$ or $m_{Z}^{2}$, namely, the case for the $h \rightarrow \gamma \gamma$ or $h \rightarrow Z \gamma$ decay, we can also check $\Gamma_{h V \gamma}^{1}=p \cdot q \Gamma_{h V \gamma}^{2}$ even for $W$-boson loops.

\section{B. Results at $\sqrt{s}=250 \mathrm{GeV}$}

We show numerical results for the cross sections at $\sqrt{s}=$ $250 \mathrm{GeV}$ in the IDM, the ITM, and the THDM, in order. We also discuss correlations between the $e^{+} e^{-} \rightarrow h \gamma$ production and the $h \rightarrow \gamma \gamma$ decay as well as the $h \rightarrow Z \gamma$ decay. To see deviations from the predictions in the SM, we evaluate the ratios of the total cross sections and the partial decay rates

$$
\begin{gathered}
\Delta R\left(e^{+} e^{-} \rightarrow h \gamma\right)=\frac{\sigma_{\mathrm{NP}}\left(e^{+} e^{-} \rightarrow h \gamma\right)}{\sigma_{\mathrm{SM}}\left(e^{+} e^{-} \rightarrow h \gamma\right)}-1\left(\equiv \Delta R_{h \gamma}\right), \\
\Delta R(h \rightarrow V \gamma)=\frac{\Gamma_{\mathrm{NP}}(h \rightarrow V \gamma)}{\Gamma_{\mathrm{SM}}(h \rightarrow V \gamma)}-1\left(\equiv \Delta R_{V \gamma}\right),
\end{gathered}
$$



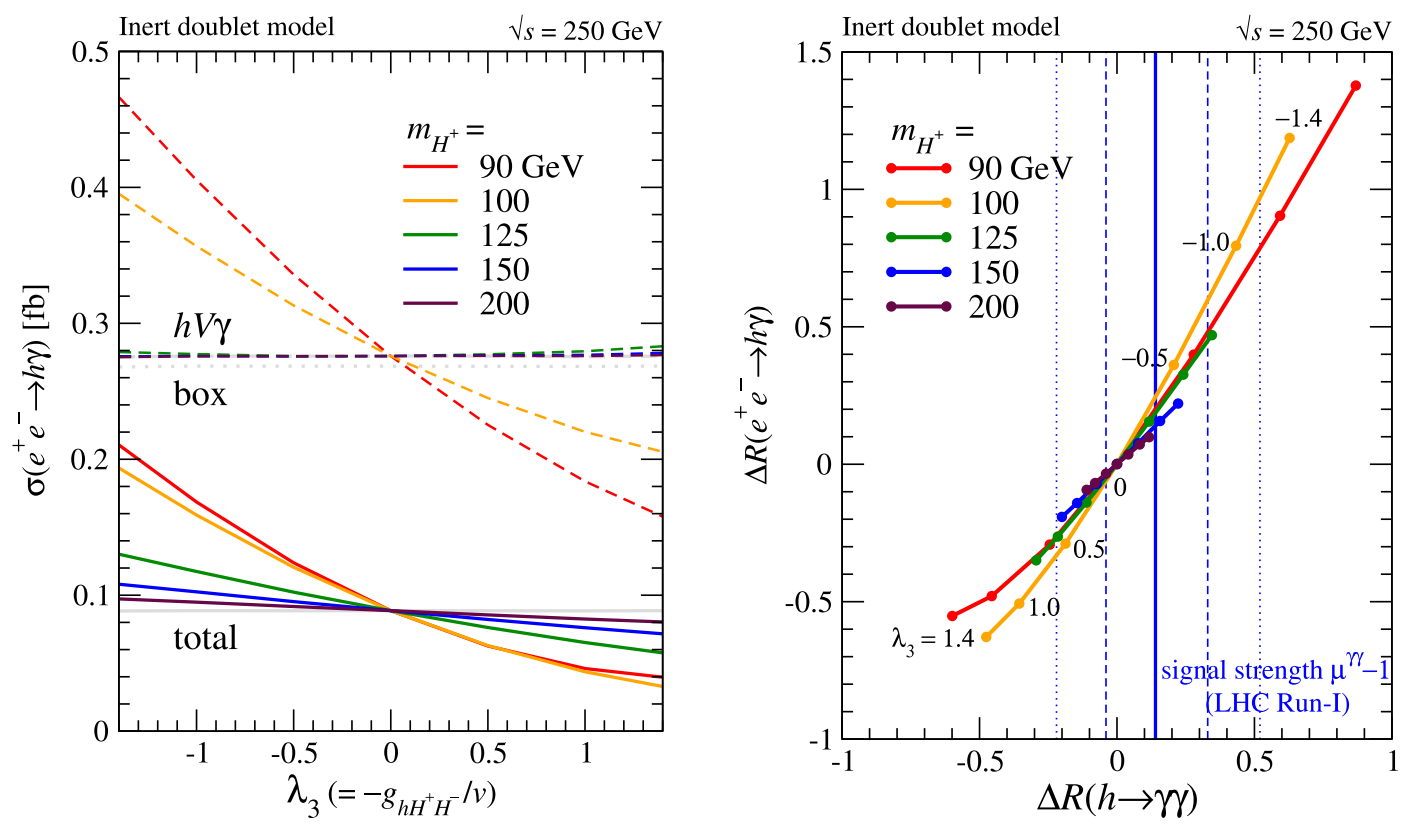

FIG. 5. (Left) Total cross sections for $e^{+} e^{-} \rightarrow h \gamma$ in the IDM as a function of $\lambda_{3}$ at $\sqrt{s}=250 \mathrm{GeV}$ with different charged Higgs-boson masses. The contributions only from the $h V \gamma$ (dashed lines) or box (dotted) diagrams are also shown as a reference. Gray lines denote the SM prediction. (Right) Correlations between $\Delta R(h \rightarrow \gamma \gamma)$ and $\Delta R\left(e^{+} e^{-} \rightarrow h \gamma\right)$, defined in Eqs. (35) and (34). Vertical blue lines indicate the $h \rightarrow \gamma \gamma$ signal strength from the LHC Run-I data with the $1 \sigma$ and $2 \sigma$ uncertainties.

where $V=\gamma$ or $Z$ and $\sigma_{\mathrm{NP}}$ and $\Gamma_{\mathrm{NP}}$ are evaluated in each extended Higgs model.

\section{IDM}

In the IDM, the relevant parameters for the $e^{+} e^{-} \rightarrow h \gamma$ process are the charged Higgs-boson mass $m_{H^{+}}$and the $h-H^{+}-H^{-}$coupling $g_{h H^{+} H^{-}}$in Eq. (7). We choose $m_{H^{+}}$ and $\lambda_{3}$ as free parameters for our illustration, while we fix the other parameters, $m_{H}, m_{A}$, and $\lambda_{2}$, so as to avoid the constraints discussed in Sec. III A. ${ }^{10}$ We note that our results agree with the previous study in Ref. [19].

In Fig. 5 (left), we show total cross sections for $e^{+} e^{-} \rightarrow h \gamma$ in the IDM as a function of $\lambda_{3}$. In addition to the total cross sections (solid lines), we separately show the triangle $h V \gamma$ (dashed) and box (dotted) contributions as a reference. Horizontal gray lines denote the SM prediction. In the IDM, as shown in Eq. (6), the SM-like Higgs-boson couplings to SM fermions and weak bosons are identical to the SM ones. Therefore, the box contributions are same as in the SM. On the other hand, the top and $W$ loops in the $h V \gamma$ contributions are also same as in the SM, but the charged scalar loops give additional contributions and modify the total rate if $g_{\mathrm{hH}^{+} \mathrm{H}^{-}}$is nonzero and the charged Higgs boson is light enough. At $\sqrt{s}=250 \mathrm{GeV}$, if $m_{H^{+}}<125 \mathrm{GeV}$, i.e., above the $\mathrm{H}^{+} \mathrm{H}^{-}$pair threshold, the amplitudes of the

\footnotetext{
${ }^{10}$ For instance, with $m_{H}=62.5 \mathrm{GeV}, m_{H^{+}}=m_{A}=100 \mathrm{GeV}$, and $\lambda_{2}=8.25$, the range $\lambda_{3} \gtrsim-1.2$ is allowed. We note that such a parameter region also satisfies dark matter constraints $[34,42]$.
}

charged scalar loops are complex, while those are real for $m_{H^{+}} \geq 125 \mathrm{GeV}$; see Fig. 11 in the Appendix. This explains the quantitative difference for the deviations between the cases below and above $m_{H^{+}}=\sqrt{s} / 2=125 \mathrm{GeV}$.

When $\lambda_{3}$ is negative (positive), the production is enhanced (reduced) from the SM prediction. Because of $\lambda_{3} \gtrsim-1.4$ for $\lambda_{2} \sim 8$ from the theoretical constraints [see Fig. 3 (left)] and because of the exclusion bound of $m_{H^{+}}$ from the null result of the direct search at the LEP, as discussed in Sec. III A, a possible largest enhancement in the IDM is about 2.5 times the SM prediction.

The same triangle $H^{ \pm}$loops appear in the $h \rightarrow \gamma \gamma$ process, which can modify the observed Higgs decay rate. One can find the explicit partial decay rate in the IDM in Refs. [27,8688], which is implemented in H-COUP [25]. In Fig. 5 (right), we show correlations between $\Delta R_{\gamma \gamma}$ and $\Delta R_{h \gamma}$, defined in Eqs. (35) and (34), respectively. There are clear positive correlations. As the $e^{+} e^{-} \rightarrow h \gamma$ process is more intricate than $h \rightarrow \gamma \gamma$, namely, the off-shell-ness of one of the photons and the additional $h Z \gamma$ and box contributions, the slope of the correlations differs by the charged Higgs-boson mass; $\left|\Delta R_{h \gamma}\right|>\left|\Delta R_{\gamma \gamma}\right|$ for $m_{H^{+}}<150 \mathrm{GeV}$, while $\Delta R_{h \gamma} \sim \Delta R_{\gamma \gamma}$ for $m_{H^{+}} \gtrsim 150 \mathrm{GeV}$.

In Fig. 5 (right), we also present the $h \rightarrow \gamma \gamma$ signal strength from the LHC Run-I data with the $1 \sigma$ and $2 \sigma$ uncertainties, $\mu^{\gamma \gamma}=1.14_{-0.18}^{+0.19}$ [89], by vertical blue lines. This provides the stronger constraints for light charged Higgs bosons. For instance, for the case of $m_{H^{+}}=100 \mathrm{GeV},-1.2 \lesssim \lambda_{3} \lesssim$ 0.6 is allowed at $95 \%$ C.L. If we observe $\Delta R_{\gamma \gamma} \sim 0$ with 

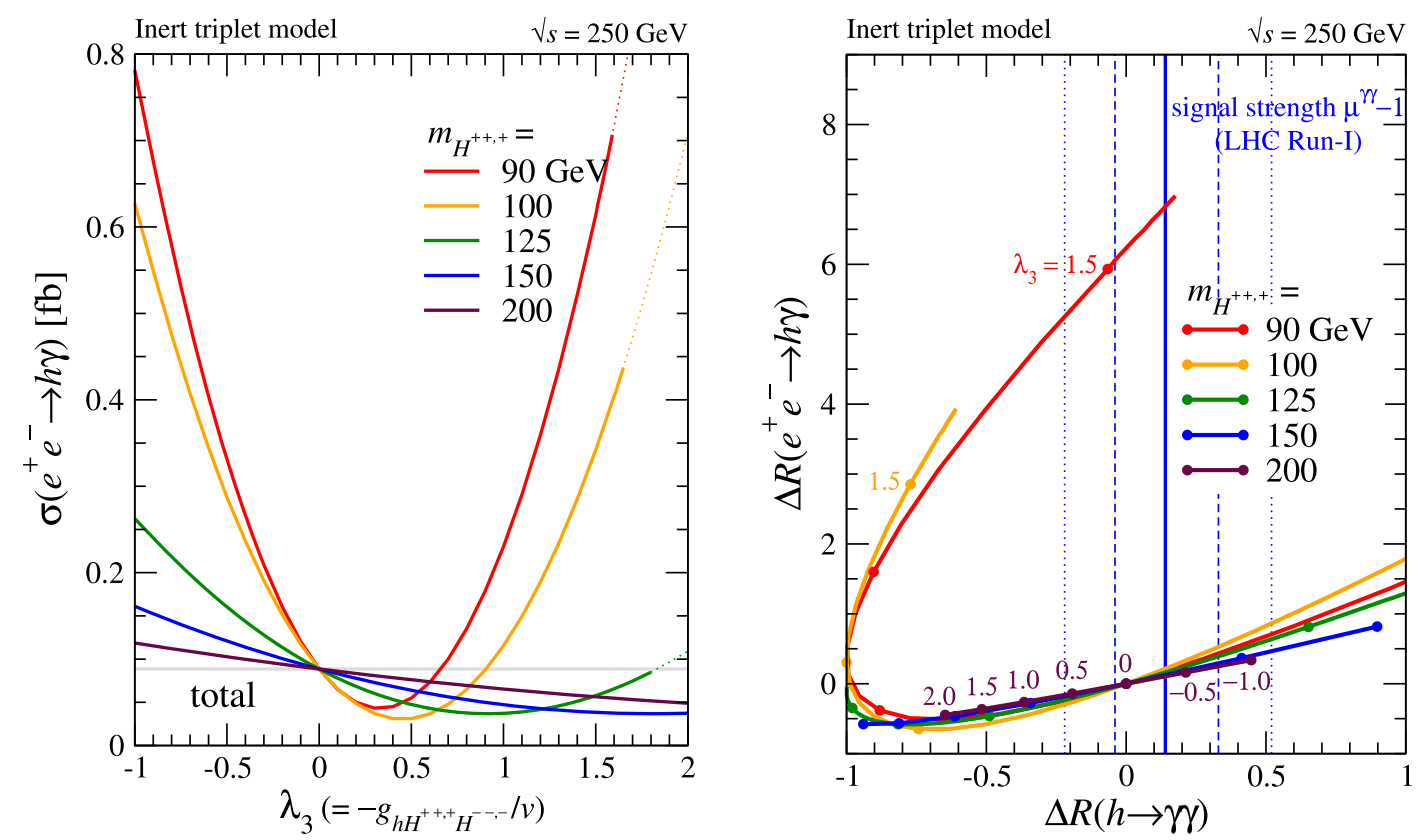

FIG. 6. (Left) Total cross sections for $e^{+} e^{-} \rightarrow h \gamma$ in the ITM as a function of $\lambda_{3}$ at $\sqrt{s}=250 \mathrm{GeV}$ with different charged Higgs-boson masses. The right regions shown by dotted lines for $m_{H^{++}}=m_{H^{+}}=90,100,125 \mathrm{GeV}$ indicate parameter regions excluded by the requirement of the existence of the inert vacuum. A gray line denotes the SM prediction. (Right) Correlations between $\Delta R(h \rightarrow \gamma \gamma)$ and $\Delta R\left(e^{+} e^{-} \rightarrow h \gamma\right)$. Vertical blue lines indicate the $h \rightarrow \gamma \gamma$ signal strength from the LHC Run-I data with the $1 \sigma$ and $2 \sigma$ uncertainties.

smaller uncertainties in future experiments, the parameter region of large $\left|\lambda_{3}\right|$ and small $m_{H^{+}}$will be further constrained.

\section{ITM}

Turn to the ITM. For simplicity, we assume $m_{H^{++}}=m_{H}$ (or $\lambda_{5}=0$ ). Under this assumption, $g_{h H^{++} H^{--}}=g_{h H^{+} H^{-}}=$ $-v \lambda_{3}$ and $m_{H^{+}}=m_{H^{++}}$, and hence the situation is very similar to the IDM, except for additional loops of the doubly charged scalars.

Similarly to Fig. 5 (left) in the IDM, we show total cross sections in the ITM as a function of $\lambda_{3}$ in Fig. 6 (left). The production rate is highly enhanced due to the considerable contributions from the doubly charged scalars. This is simply because photons couple to particles with the electromagnetic charge. The vertex functions $\Gamma_{h \gamma \gamma}^{1,2}$ in Eqs. (A6) and (A7) are proportional to $\sum_{S} Q_{S}^{2}$, and hence, for the $\lambda_{5}=0$ case, the contribution from the charged scalar loops of the $h \gamma \gamma$ vertex in the ITM is five times larger than that in the IDM at the amplitude level.

A remarkable difference from the IDM is that, even for positive $\lambda_{3}$, the production rate can be significantly enhanced if the charged Higgs bosons are light. This is because the charged scalar contributions overwhelm all the other contributions. A caveat is that there is an upper bound on $\lambda_{3}$ from the requirement of the existence of the inert vacuum, which depends on the masses of the inert scalars, as shown in Fig. 3 (right). The excluded regions are indicated by dotted lines. We also note that, even in the IDM, we naively expect that the cross section could be enhanced if we take much larger positive $\lambda_{3}$. However, again, such parameter region is not allowed by the theoretical constraints.

In Fig. 6 (right), we show correlations between $\Delta R_{\gamma \gamma}$ and $\Delta R_{h \gamma}$. The $h \rightarrow \gamma \gamma$ decay with doubly charged scalars has been studied in Refs. [48,58,90-96] and was newly implemented in H-COUP. The lower part of the plot $\left(\Delta R_{h \gamma}<2\right)$ is qualitatively similar to Fig. 5 (right) in the IDM. Because of the huge enhancement (reduction) of the production and the decay by the doubly charged scalars, the LHC $h \rightarrow \gamma \gamma$ signal strength provides more severe constraints on the masses and the couplings than in the IDM. For instance, for the case of $m_{H^{++}}=m_{H^{+}}=$ $100(200) \mathrm{GeV},-0.2(-1.1) \lesssim \lambda_{3} \lesssim 0.1(0.6)$ is allowed at $95 \%$ C.L.

Again, a remarkable difference from the IDM is that we can find a particular parameter region where $\sigma\left(e^{+} e^{-} \rightarrow h \gamma\right)$ can be significantly enhanced by a factor of $6-8$, but the $\Gamma(h \rightarrow \gamma \gamma)$ still remains as in the SM; e.g., the case for $m_{H^{++}}=m_{H^{+}}=90 \mathrm{GeV}$ with $1.4 \lesssim \lambda_{3} \lesssim 1.6$ is allowed at 95\% C.L. under the theoretical and current experimental constraints. Such parameter regions are quite attractive for future $e^{+} e^{-}$colliders, in which we can expect a synergy between the observation of the rare $h \gamma$ production and the direct discovery of charged Higgs bosons.

\section{THDM}

In the THDM with softly broken $Z_{2}$ symmetry, the couplings of the SM-like Higgs boson with SM fermions 

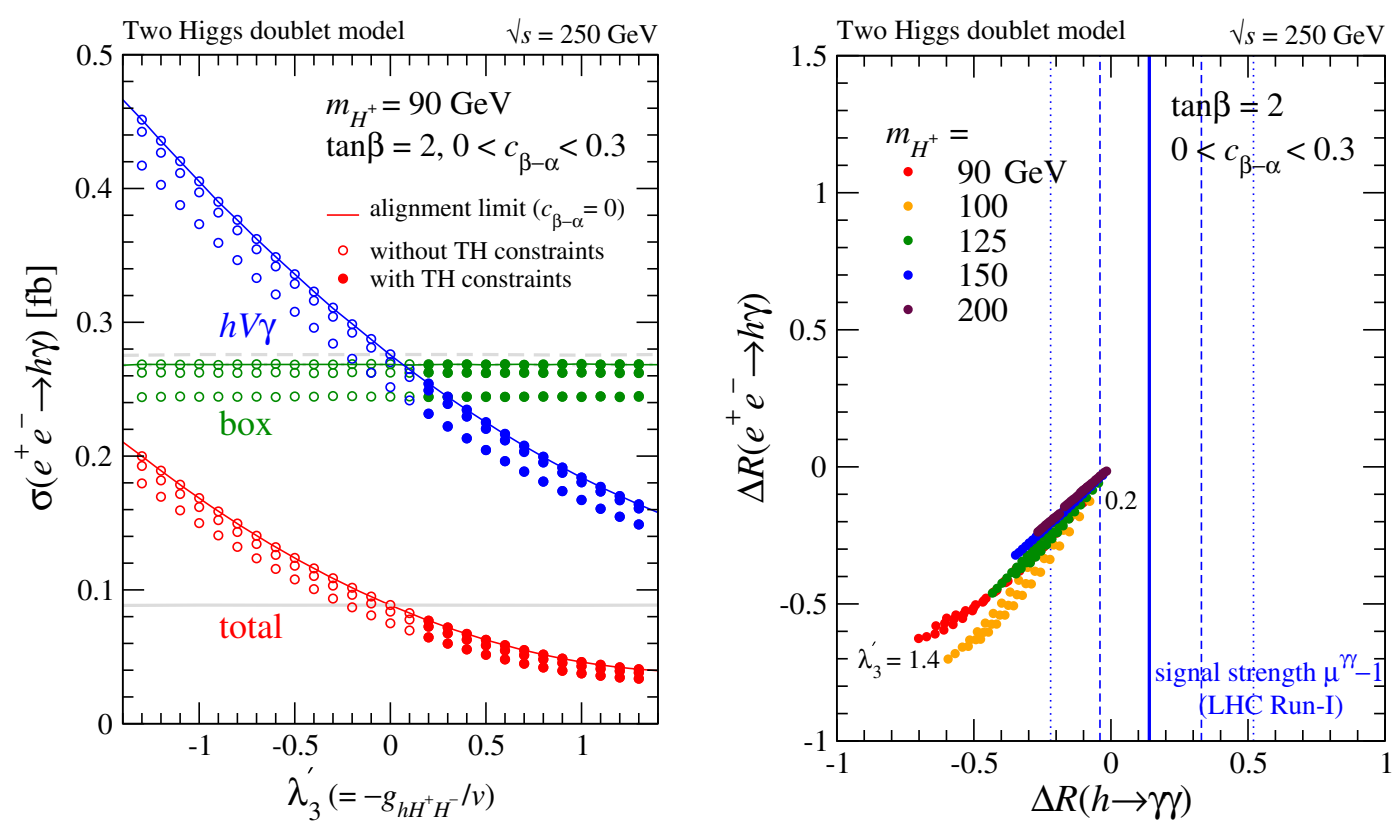

FIG. 7. (Left) Total cross sections for $e^{+} e^{-} \rightarrow h \gamma$ in the THDM as a function of $\lambda_{3}$ at $\sqrt{s}=250 \mathrm{GeV}$ for $m_{H^{+}}=90 \mathrm{GeV}$, where we fix $\tan \beta=2$ and vary $c_{\beta-\alpha}$ from 0 to 0.3 . Gray lines denote the SM prediction. (Right) Correlations between $\Delta R(h \rightarrow \gamma \gamma)$ and $\Delta R\left(e^{+} e^{-} \rightarrow h \gamma\right)$. Vertical blue lines indicate the $h \rightarrow \gamma \gamma$ signal strength from the LHC Run-I data with the $1 \sigma$ and $2 \sigma$ uncertainties.

and weak bosons are modified through the mixing with other neutral scalars, as shown in Eq. (25). For $c_{\beta-\alpha}=0$, the so-called alignment limit, there is no mixing effect; i.e., $\kappa_{f}=\kappa_{V}=1$. Therefore, if we take the same value of $g_{h H^{+} H^{-}}$in Eq. (26), i.e., $\lambda_{3}^{\prime}=\lambda_{3}$, the prediction in the THDM is identical to that in the IDM, which is explicitly shown for $m_{H^{+}}=90 \mathrm{GeV}$ by solid lines in Fig. 7 (left).

Once $c_{\beta-\alpha}$ deviates from 0 , the scaling factors $\kappa_{f}$ and $\kappa_{V}$ deviate from 1 . To see this mixing effect, we take $\tan \beta=2$ and vary $c_{\beta-\alpha}$ from 0 to 0.3 , which is allowed in the current constraints from the Higgs coupling measurements [97,98]. We note that a negative $c_{\beta-\alpha}$ is also allowed, and the results are very similar to the positive case. Unlike the inert models, not only the $h V \gamma$ contributions but also the box contributions deviate from the SM due to the scaling factors of the SM-like Higgs couplings. Therefore, even for $\lambda_{3}^{\prime}=0$, the cross section in the THDM differs from that in the SM if $c_{\beta-\alpha} \neq 0$. Except such small mixing effects, the qualitative behaviors are very similar to the case in the IDM, namely, negative (positive) $\lambda_{3}^{\prime}$ can enhance (reduce) the production rate.

As shown in filled circles in Fig. 7 (left), however, the theoretical constraints in the THDM are much more severe than those in the IDM; the vacuum stability requires $\lambda_{3}^{\prime}>$ 0.1 for $\tan \beta>2$ (see Fig. 4). As mentioned in Sec. III C, although such light charged Higgs bosons with $\tan \beta=2$ are allowed in the type-I and type-X models for the $B$-physics experiments [78], the current LHC data require higher $\tan \beta$ values to avoid the lower limit on the mass, $m_{H^{+}}>160 \mathrm{GeV}$ [77]. On the other hand, for larger $\tan \beta$, the allowed region with the theoretical constraints in Fig. 7 (left) becomes smaller due to the unitarity constraint. ${ }^{11}$ Therefore, in the THDMs, there is a tension between the allowed parameter region and the preferred region to observe large deviations in the $e^{+} e^{-} \rightarrow h \gamma$ signal.

In Fig. 7 (right), we present correlations between $\Delta R_{\gamma \gamma}$ and $\Delta R_{h \gamma}$, where we still keep $\tan \beta=2$ as an illustration. Although we vary $c_{\beta-\alpha}$ as $0<c_{\beta-\alpha}<0.3$, this mixing effect is small, and hence the correlations look similar to ones in the IDM in Fig. 5 (right), except for no allowed parameter region for positive $\Delta R_{\gamma \gamma}$ and $\Delta R_{h \gamma}$. In short, the production rate in the THDM tends to reduce from the SM prediction after imposing the theoretical constraints, and the magnitude of the deviation is minor after imposing all the current experimental constraints.

Before turning to the case for $\sqrt{s}=500 \mathrm{GeV}$, we mention the $h \rightarrow Z \gamma$ decay, which is closely related to the $h \rightarrow \gamma \gamma$ decay. As seen in Fig. $8, \Delta R_{\gamma \gamma}$ and $\Delta R_{Z \gamma}$ are strongly correlated in the IDM and the ITM. The correlation in the THDM is very similar to that in the IDM, and hence we omit it. The sensitivity to the charged scalar loops for the $h \rightarrow Z \gamma$ decay is much weaker than for the $h \rightarrow \gamma \gamma$ decay. Moreover, from the experimental point of view, the observation of the $h \rightarrow Z_{\gamma}$ decay is much more difficult than the $h \rightarrow \gamma \gamma$ decay; the current LHC data only give the upper limit on the production cross section times the branching ratio for $p p \rightarrow h \rightarrow Z \gamma$ as about seven times

\footnotetext{
${ }^{11}$ For instance, for $m_{H^{+}}=90 \mathrm{GeV}$ with $\tan \beta=8$, which is still allowed by experiments in the type-I THDM, $\lambda_{3}^{\prime}$ is constrained between 0.3 and 0.5 by the vacuum stability and the unitarity; see also Fig. 4.
} 

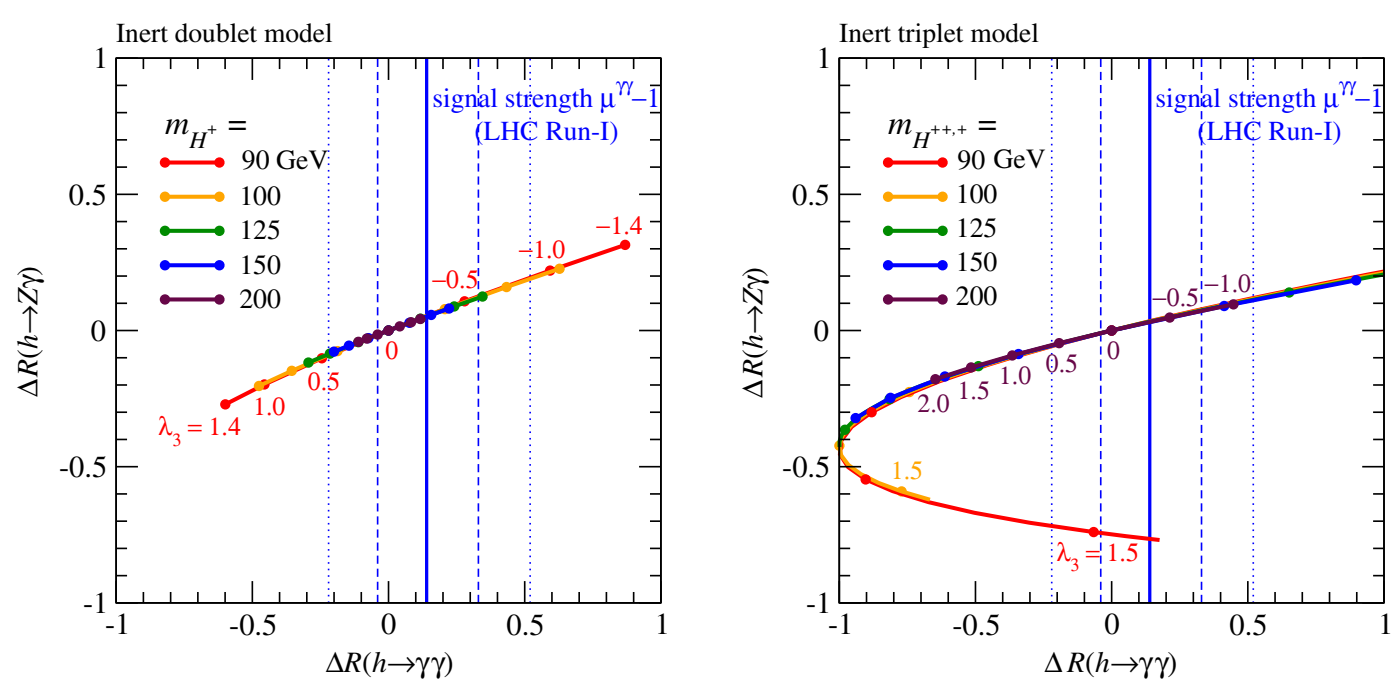

FIG. 8. Correlations between $\Delta R(h \rightarrow \gamma \gamma)$ and $\Delta R(h \rightarrow Z \gamma)$ in the IDM (left) and in the ITM (right).
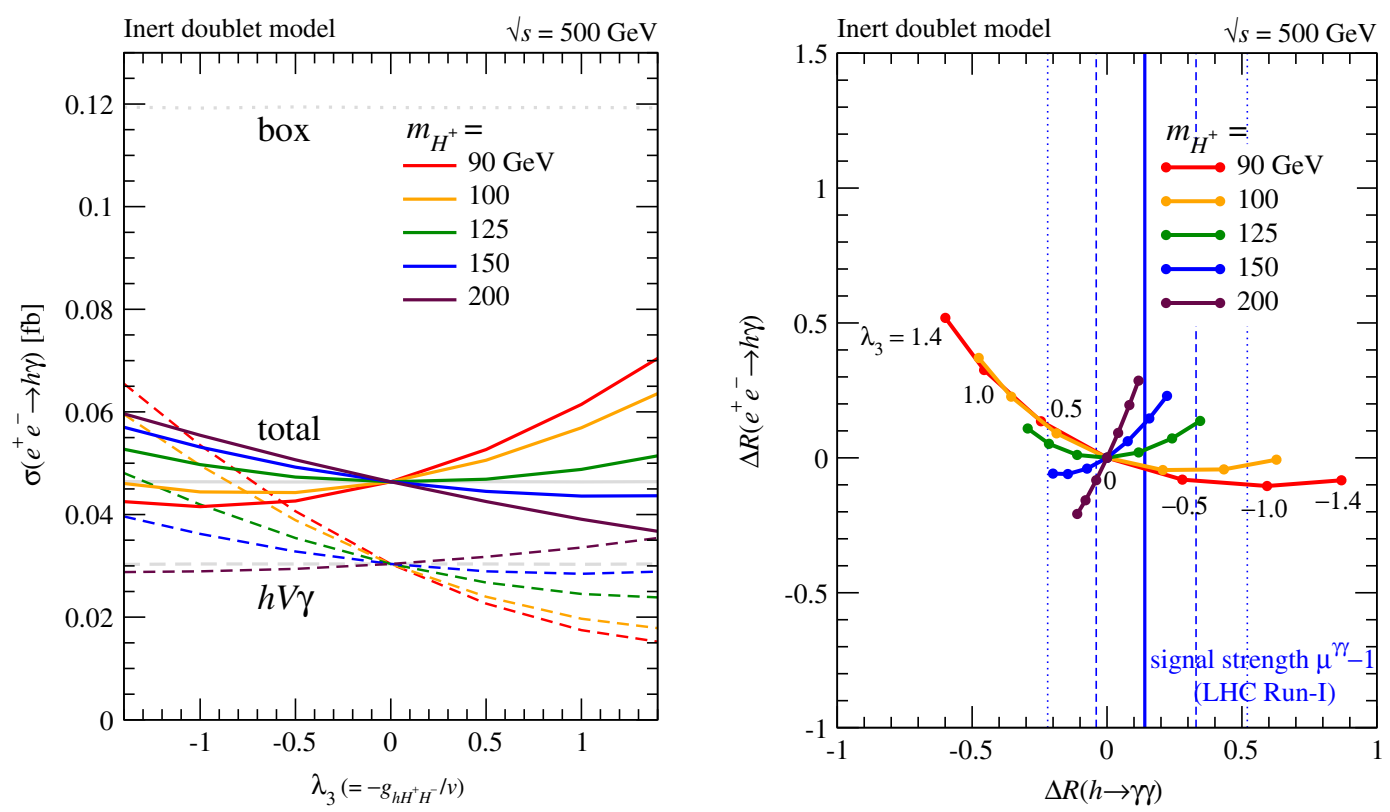

FIG. 9. Same as Fig. 5, but at $\sqrt{s}=500 \mathrm{GeV}$. Total cross sections for $e^{+} e^{-} \rightarrow h \gamma$ as a function of $\lambda_{3}$ (left) and correlations between $\Delta R(h \rightarrow \gamma \gamma)$ and $\Delta R\left(e^{+} e^{-} \rightarrow h \gamma\right)$ (right) in the IDM.

the SM prediction $[99,100]$. An interesting thing in the ITM is that, for the case of $m_{H^{+}}=m_{H^{++}}=90 \mathrm{GeV}$ with $\lambda_{3}=1.5$, leading to the large enhancement for the $h \gamma$ production, we can retain the $h \rightarrow \gamma \gamma$ decay as in the SM, but the $h \rightarrow Z \gamma$ decay should be suppressed by about $70 \%$ from the SM prediction. In other words, if we observe the $h \rightarrow Z \gamma$ decay as in the SM in future experiments, we could not expect such $h \gamma$ enhancement.

\section{Results at $\sqrt{\mathrm{s}}=\mathbf{5 0 0} \mathrm{GeV}$}

As we have seen in Fig. 1, the $e^{+} e^{-} \rightarrow h \gamma$ process in the $\mathrm{SM}$ has the second peak around $\sqrt{s}=500 \mathrm{GeV}$, the cross section of which is about a half of that at $\sqrt{s}=250 \mathrm{GeV} .^{12}$ Here, we repeat the exact same analyses in the previous subsection but consider the collision energy at $500 \mathrm{GeV}$, where one can find rather different parameter dependences of the cross sections and the $\Delta R_{\gamma \gamma}-\Delta R_{h \gamma}$ correlations below.

In Fig. 9, similar to Fig. 5, we show $\sigma\left(e^{+} e^{-} \rightarrow h \gamma\right)$ as a function of $\lambda_{3}$ (left panel) and $\Delta R_{\gamma \gamma}-\Delta R_{h \gamma}$ (right panel) in the IDM at $\sqrt{s}=500 \mathrm{GeV}$. Unlike the case at $\sqrt{s}=250 \mathrm{GeV}$, the production rate can be enhanced for

\footnotetext{
${ }^{12}$ The $K$ factor of the next-to-leading-order QCD corrections is about $-0.4 \%$ and $14 \%$ at $\sqrt{s}=250$ and $500 \mathrm{GeV}$, respectively [9].
} 

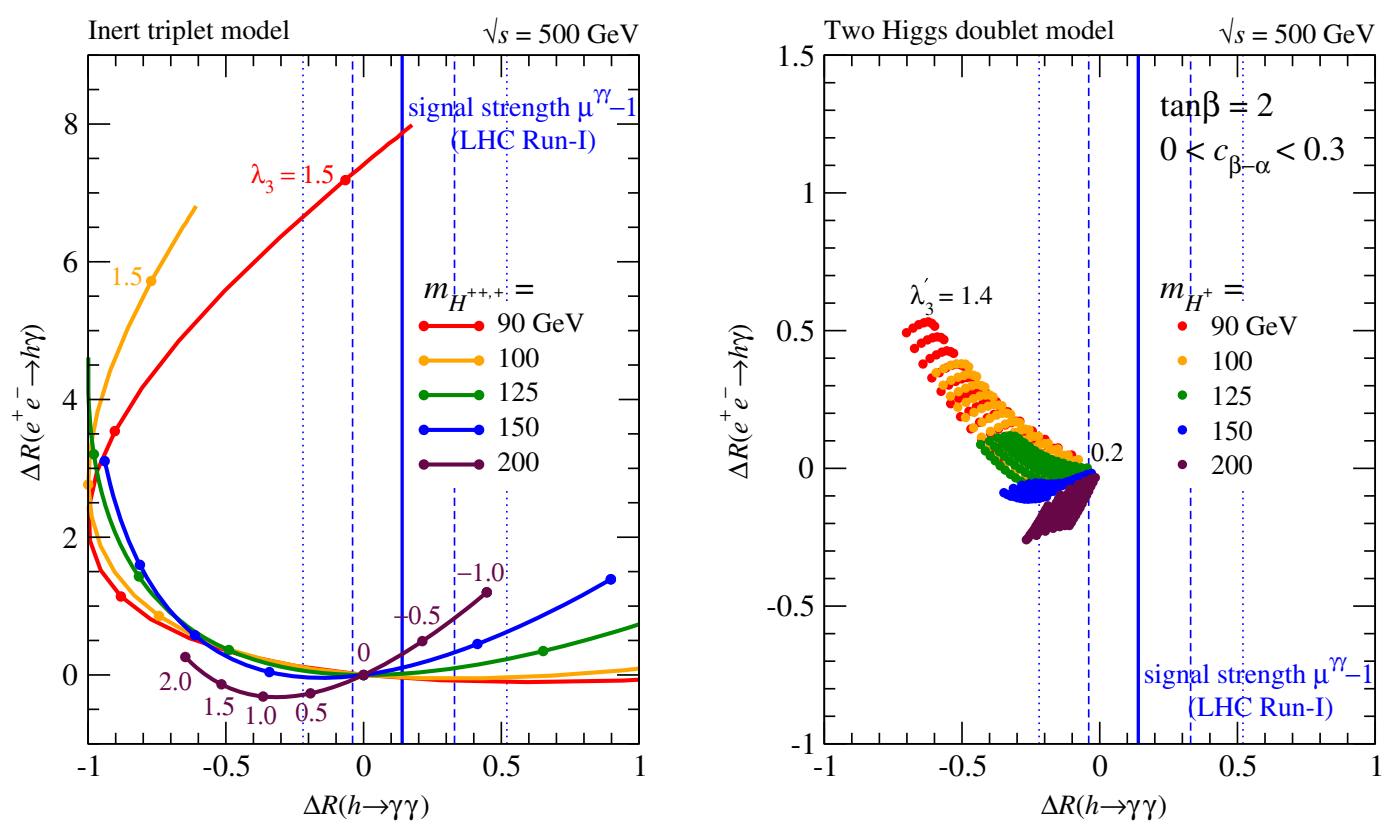

FIG. 10. Correlations between $\Delta R(h \rightarrow \gamma \gamma)$ and $\Delta R\left(e^{+} e^{-} \rightarrow h \gamma\right)$ in the ITM (left) and in the THDM (right) at $\sqrt{s}=500 \mathrm{GeV}$.

$m_{H^{+}} \geq 125 \mathrm{GeV}$ with negative $\lambda_{3}$ as well as for $m_{H^{+}} \leq$ $125 \mathrm{GeV}$ with positive $\lambda_{3}$. Although the $\lambda_{3}$ dependence of the cross sections from the $h V \gamma$ diagrams (dashed lines) is similar to the case at $\sqrt{s}=250 \mathrm{GeV}$, i.e., the $h V \gamma$ contributions are enhanced (reduced) for negative (positive) $\lambda_{3}$ for light charged scalars, the contributions of each amplitude are rather different from the $\sqrt{s}=250 \mathrm{GeV}$ case, resulting in the different $\lambda_{3}$ dependence of the total cross sections (solid lines). At $\sqrt{s}=500 \mathrm{GeV}$, the box contributions (a gray dotted line) are dominant (see also Fig. 1), and the top-loop contributions are also significant. Moreover, all the particles in the loops, including charged scalars of our benchmark, can be on shell; i.e., all the amplitudes become complex, leading to intricate interference effects. Since the Higgs-boson decay does not depend on $\sqrt{s}$, we find rather different correlations between $\Delta R_{\gamma \gamma}$ and $\Delta R_{h \gamma}$ for different masses of the charged scalars. Especially, the correlations at $\sqrt{s}=250$ and $500 \mathrm{GeV}$ are opposite for $m_{H^{+}}=90-100 \mathrm{GeV}$.

Figure 10 presents correlations $\Delta R(h \rightarrow \gamma \gamma)$ and $\Delta R\left(e^{+} e^{-} \rightarrow h \gamma\right)$ in the ITM (left panel) and in the THDM (right panel) at $\sqrt{s}=500 \mathrm{GeV}$. All the behaviors can be understood from the discussions above. We stress that in the ITM we can find a particular parameter region where the $h \gamma$ production is enhanced by a factor of 7.5-9, but the $h \rightarrow \gamma \gamma$ decay still remains as in the SM, if the mass of the charged Higgs boson is around $90 \mathrm{GeV}$. Similarly to the $\sqrt{s}=250 \mathrm{GeV}$ case, such parameter region predicts the suppression of the $h \rightarrow Z \gamma$ decay.

\section{SUMMARY}

We studied Higgs production associated with a photon at $e^{+} e^{-}$colliders in the IDM, the ITM, and the THDM as distinctive new physics benchmark scenarios. The cross section in the SM is maximal around $\sqrt{s}=250 \mathrm{GeV}$, and we presented how and how much the new physics can enhance or reduce the production rate. We also discussed the correlations with the $h \rightarrow \gamma \gamma$ and $h \rightarrow Z \gamma$ decay rates. We found that, with a sizable coupling to a SM-like Higgs boson, charged scalars can give considerable contributions to both the production and the decay if their masses are around $100 \mathrm{GeV}$. Under the theoretical constraints from vacuum stability and perturbative unitarity as well as the current constraints from the Higgs measurements at the LHC, the production rate can be enhanced from the SM prediction at most by a factor of 2 in the IDM. In the ITM, in addition, we found a particular parameter region, $m_{H^{++}}=m_{H^{+}} \sim$ $90 \mathrm{GeV}$ with $\lambda_{3} \sim 1.5$, where the $h \gamma$ production significantly increases by a factor of about 6 to 8 , but the $h \rightarrow \gamma \gamma$ decay still remains as in the SM. For such a parameter region, on the other hand, the $h \rightarrow Z \gamma$ decay is suppressed. In the THDM, possible deviations from the SM prediction are minor in the viable parameter space. We also showed that the dependence of the cross section on the model parameters is rather different at $\sqrt{s}=500 \mathrm{GeV}$, leading to the possibility of accessing more information on the Higgs sector.

As a final remark, we would like to point out that new physics can affect not only the total cross section but also the differential distribution, which would be important for realistic simulation of the signal events to compare with the background.

\section{ACKNOWLEDGMENTS}

The work of S. K. is supported in part by Grant-in-Aid for Scientific Research on Innovative Areas, the Ministry of 
Education, Culture, Sports, Science and Technology, Grants No. 16H06492 and No. 18H04587 and Grant H2020-MSCA-RISE-2014 No. 645722 (Non-Minimal Higgs). The work of K. M. was supported in part by JSPS KAKENHI Grant No. 18K03648. The work of K. S. was supported in part by JSPS KAKENHI Grant No. $18 \mathrm{~J} 12866$.

\section{APPENDIX: $h \gamma \gamma$ AND $h z \gamma$ VERTEX FUNCTIONS}

In this Appendix, we present analytical expressions for the $h \gamma \gamma$ and $h Z \gamma$ vertex functions at one-loop level in extended Higgs models.
The $h \gamma \gamma$ vertex is loop induced and does not need to be renormalized, so the vertex functions $\Gamma_{h \gamma \gamma}^{i}(i=1,2)$ directly correspond to its one-particle irreducible (1PI) diagram contributions

$$
\Gamma_{h \gamma \gamma}^{i}\left(p_{1}^{2}, p_{2}^{2}, p_{h}^{2}\right)=\Gamma_{h \gamma \gamma}^{i, 1 \mathrm{PI}}\left(p_{1}^{2}, p_{2}^{2}, p_{h}^{2}\right) .
$$

The 1PI diagram contributions are divided into fermion loop, $W$-boson loop, and charged scalar loop contributions, which are expressed in terms of Passarino-Veltman functions [101]. ${ }^{13}$ Regardless of a choice of models, each contribution is expressed as

$$
\begin{aligned}
& \Gamma_{h \gamma \gamma}^{1,1 \mathrm{PI}}\left(p_{1}^{2}, p_{2}^{2}, p_{h}^{2}\right)_{F}=-\kappa_{f} \frac{e^{2} g m_{W}}{16 \pi^{2}} \sum_{f} 4 N_{c} Q_{f}^{2} \frac{m_{f}^{2}}{m_{W}^{2}}\left\{\frac{1}{2}\left(p_{1}^{2}+p_{2}^{2}-p_{h}^{2}\right) C_{0}+4 C_{3}\right\}(f, f, f), \\
& \Gamma_{h \gamma \gamma}^{2,1 \mathrm{PI}}\left(p_{1}^{2}, p_{2}^{2}, p_{h}^{2}\right)_{F}=-\kappa_{f} \frac{e^{2} g m_{W}}{16 \pi^{2}} \sum_{f} 4 N_{c} Q_{f}^{2} \frac{m_{f}^{2}}{m_{W}^{2}}\left(C_{0}+4 C_{2}\right)(f, f, f), \\
& \Gamma_{h \gamma \gamma}^{1,1 \mathrm{PI}}\left(p_{1}^{2}, p_{2}^{2}, p_{h}^{2}\right)_{V}=\kappa_{V} \frac{e^{2} g m_{W}}{16 \pi^{2}}\left\{\left(5 p_{1}^{2}+5 p_{2}^{2}-7 p_{h}^{2}-m_{h}^{2}\right) C_{0}+4\left(6+\frac{m_{h}^{2}}{m_{W}^{2}}\right) C_{3}\right\}(W, W, W), \\
& \Gamma_{h \gamma \gamma}^{2,1 \mathrm{PI}}\left(p_{1}^{2}, p_{2}^{2}, p_{h}^{2}\right)_{V}=\kappa_{V} \frac{4 e^{2} g m_{W}}{16 \pi^{2}}\left\{4 C_{0}+\left(6+\frac{m_{h}^{2}}{m_{W}^{2}}\right) C_{2}\right\}(W, W, W),
\end{aligned}
$$

$\Gamma_{h \gamma \gamma}^{1,1 \mathrm{PI}}\left(p_{1}^{2}, p_{2}^{2}, p_{h}^{2}\right)_{S}=-\frac{8 e^{2}}{16 \pi^{2}} \sum_{S} g_{h S S^{*}} Q_{S}^{2} C_{3}(S, S, S)$,

$\Gamma_{h \gamma \gamma}^{2,1 \mathrm{PI}}\left(p_{1}^{2}, p_{2}^{2}, p_{h}^{2}\right)_{S}=-\frac{8 e^{2}}{16 \pi^{2}} \sum_{S} g_{h S S^{*}} Q_{S}^{2} C_{2}(S, S, S)$,

where $C_{2}$ and $C_{3}$ are defined by

$$
\begin{aligned}
& C_{2}(X, X, X)=C_{12}(X, X, X)+C_{23}(X, X, X), \\
& C_{3}(X, X, X)=C_{24}(X, X, X)-\frac{1}{4} B_{0}\left(p_{h}^{2} ; X, X\right) .
\end{aligned}
$$

The scaling factors $\kappa_{f, V}$ and the trilinear couplings between the SM-like Higgs boson and charged scalars $g_{h S S^{*}}$ depend on each extended Higgs model. See Eqs. (6) and (7) in the

\footnotetext{
${ }^{13}$ For convenience, we abbreviate $C$ and $B$ functions as $C_{i}\left(p_{1}^{2}, p_{2}^{2}, p_{h}^{2} ; m_{X}, m_{Y}, m_{Z}\right) \equiv C_{i}(X, Y, Z)$ and $B_{i}\left(p^{2} ; m_{X}, m_{Y}\right) \equiv$ $B_{i}\left(p^{2} ; X, Y\right)$, respectively.
}

IDM, Eqs. (14), (15) and (16) in the ITM, and Eqs. (25) and (26) in the THDM.

The $h Z \gamma$ vertex is also loop induced and needs counterterms to remove ultraviolet divergence unlike the $h \gamma \gamma$ vertex. Hence, the renormalized $h Z \gamma$ vertex functions $\Gamma_{h Z_{\gamma}}^{i}(i=1,2)$ are written as

$$
\Gamma_{h Z_{\gamma}}^{i}\left(p_{1}^{2}, p_{2}^{2}, p_{h}^{2}\right)=\Gamma_{h Z_{\gamma}}^{i, 1 \mathrm{PI}}\left(p_{1}^{2}, p_{2}^{2}, p_{h}^{2}\right)+\delta \Gamma_{h Z_{\gamma}}^{i} .
$$

The 1PI diagram contributions from fermion, $W$-boson, and charged scalar loops are expressed, respectively, as

$$
\begin{gathered}
\Gamma_{h Z_{\gamma}}^{1,1 \mathrm{PI}}\left(p_{1}^{2}, p_{2}^{2}, p_{h}^{2}\right)_{F} \\
=-\kappa_{f} \frac{e g g_{Z} m_{W}}{16 \pi^{2}} \sum_{f} 4 N_{c} Q_{f} v_{f} \frac{m_{f}^{2}}{m_{W}^{2}} \\
\quad \times\left\{\frac{1}{2}\left(p_{1}^{2}+p_{2}^{2}-p_{h}^{2}\right) C_{0}+4 C_{3}\right\}(f, f, f), \\
\Gamma_{h Z_{\gamma}}^{2,1 \mathrm{PI}}\left(p_{1}^{2}, p_{2}^{2}, p_{h}^{2}\right)_{F}=-\kappa_{f} \frac{e g g_{Z} m_{W}}{16 \pi^{2}} \sum_{f} 4 N_{c} Q_{f} v_{f} \frac{m_{f}^{2}}{m_{W}^{2}} \\
\times\left(C_{0}+4 C_{2}\right)(f, f, f),
\end{gathered}
$$




$$
\begin{aligned}
& \Gamma_{h Z \gamma}^{1,1 \mathrm{PI}}\left(p_{1}^{2}, p_{2}^{2}, p_{h}^{2}\right)_{V} \\
& =\kappa_{V} \frac{e g g_{Z} m_{W}}{16 \pi^{2}}\left[2 B_{0}\left(p_{2}^{2} ; W, W\right)+\left\{\left(5 p_{1}^{2}+5 p_{2}^{2}-7 p_{h}^{2}\right) c_{W}^{2}\right.\right. \\
& \left.-2 p_{1}^{2}+p_{2}^{2}+p_{h}^{2}+3 m_{W}^{2}+m_{h}^{2} s_{W}^{2}\right\} C_{0}(W, W, W) \\
& \left.+2\left\{12 c_{W}^{2}-2+\frac{m_{h}^{2}}{m_{W}^{2}}\left(2 c_{W}^{2}-1\right)\right\} C_{3}(W, W, W)\right], \\
& \Gamma_{h Z_{\gamma}}^{2,1 \mathrm{PI}}\left(p_{1}^{2}, p_{2}^{2}, p_{h}^{2}\right)_{V} \\
& =\kappa_{V} \frac{e g g_{Z} m_{W}}{16 \pi^{2}}\left[4\left(4 c_{W}^{2}-1\right) C_{0}\right. \\
& \left.+2\left\{12 c_{W}^{2}-2+\frac{m_{h}^{2}}{m_{W}^{2}}\left(2 c_{W}^{2}-1\right)\right\} C_{2}\right] \\
& \times(W, W, W), \\
& \Gamma_{h Z \gamma}^{1,1 \mathrm{PI}}\left(p_{1}^{2}, p_{2}^{2}, p_{h}^{2}\right)_{S}=-\frac{8 e g_{Z}}{16 \pi^{2}} \sum_{S} g_{h S S^{*}} Q_{S}\left(I_{3}^{S}-Q_{S} s_{W}^{2}\right) C_{3} \\
& \times(S, S, S) \text {, } \\
& \Gamma_{h Z \gamma}^{2,1 \mathrm{PI}}\left(p_{1}^{2}, p_{2}^{2}, p_{h}^{2}\right)_{S}=-\frac{8 e g_{Z}}{16 \pi^{2}} \sum_{S} g_{h S S^{*}} Q_{S}\left(I_{3}^{S}-Q_{S} s_{W}^{2}\right) C_{2} \\
& \times(S, S, S) \text {, }
\end{aligned}
$$

where $v_{f}=I_{f} / 2-Q_{f} s_{W}^{2}$ and $g_{Z}=\sqrt{g^{2}+g^{\prime 2}} \cdot Q_{S}$ and $I_{3}^{S}$ denote electric charge and isospin for charged scalars, respectively. The counterterm contributions in an improved on-shell scheme [84], which is applied in H-COUP, are written by

$\delta \Gamma_{h Z \gamma}^{1}=-\kappa_{V} \frac{e g g_{Z} m_{W}}{16 \pi^{2}} 2 B_{0}(0 ; W, W), \quad \delta \Gamma_{h Z \gamma}^{2}=0$.

We note that extra Higgs loop contributions do not appear in the counterterms. Similar to the $h \gamma \gamma$ vertex functions, $\kappa_{f}$, $\kappa_{V}$, and $g_{h S S^{*}}$ are given in each model.

For better understanding of our results in the main text, we look at the $m_{S}$ dependence of $\Gamma_{h \gamma \gamma, S}^{2}$ for the

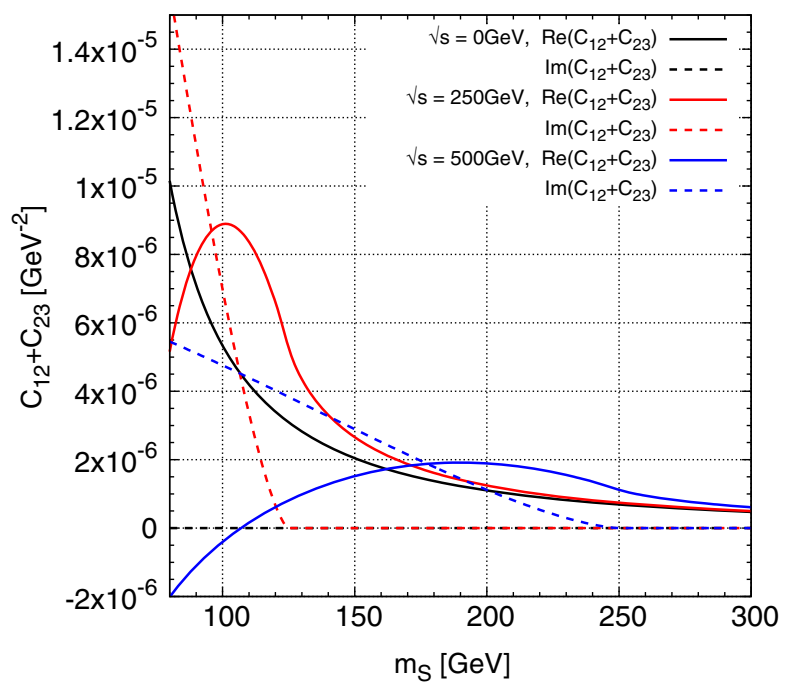

FIG. 11. Real and imaginary parts of the loop function $\left(C_{12}+\right.$ $\left.C_{23}\right)\left(p_{1}^{2}, p_{2}^{2}, p_{h}^{2} ; m_{S}, m_{S}, m_{S}\right)$ as a function of the mass of the scalar particle $m_{S}$. External momenta are fixed at $p_{1}^{2}=0$, $p_{2}^{2}=(0,250,500 \mathrm{GeV})^{2}$, and $p_{h}^{2}=m_{h}^{2}$.

$e^{+} e^{-} \rightarrow h \gamma$ production and the $h \rightarrow \gamma \gamma$ decay. As discussed in Sec. IVA, $\Gamma_{h \gamma \gamma, S}^{1}$ is related to $\Gamma_{h \gamma \gamma, S}^{2}$ due to the gauge invariance. For the kinematical configuration $p_{1}^{2}=0$, $p_{2}^{2}=s$, and $p_{h}^{2}=m_{h}^{2}, C_{2}$ can be expressed by only the $C_{0}$ and $B_{0}$ functions as

$$
\begin{aligned}
C_{2} & \left(0, s, m_{h}^{2} ; m_{S}, m_{S}, m_{S}\right) \\
& =\frac{1}{2\left(s-m_{h}^{2}\right)}\left[1+2 m_{S}^{2} C_{0}\left(0, s, m_{h}^{2} ; m_{S}, m_{S}, m_{S}\right)\right. \\
& \left.+\frac{s}{s-m_{h}^{2}}\left\{B_{0}\left(s ; m_{S}, m_{S}\right)-B_{0}\left(m_{h}^{2} ; m_{S}, m_{S}\right)\right\}\right] .
\end{aligned}
$$

In Fig. 11, we show behaviors of the loop function $C_{2}\left(\equiv C_{12}+C_{23}\right)$ as a function of the mass of scalar particle $m_{S}$, where $\sqrt{s}$ is fixed at 0,250 , and $500 \mathrm{GeV}$, denoted by black, red, and blue lines, respectively. For $m_{S}>\sqrt{s} / 2$, the loop function becomes pure real and goes to zero in the large mass limit.
[1] H. Baer, T. Barklow, K. Fujii, Y. Gao, A. Hoang, S. Kanemura et al., The international linear collider technical design report-volume 2: physics, arXiv:1306.6352.

[2] K. Fujii et al., Physics case for the $250 \mathrm{GeV}$ stage of the international linear collider, arXiv:1710.07621.

[3] S. Asai, J. Tanaka, Y. Ushiroda, M. Nakao, J. Tian, S. Kanemura et al., Report by the committee on the scientific case of the ILC operating at $250 \mathrm{GeV}$ as a Higgs Factory, arXiv:1710.08639.

[4] M. Bicer et al. (TLEP Design Study Working Group Collaboration), First look at the physics case of TLEP, J. High Energy Phys. 01 (2014) 164.

[5] C.-S.S. Group, CEPC-SPPC preliminary conceptual design report. 1. Physics and detector Reports 
No. IHEP-CEPC-DR-2015-01, IHEP-TH-2015-01, IHEPEP-2015-01.

[6] A. Abbasabadi, D. Bowser-Chao, D. A. Dicus, and W. W. Repko, Higgs photon associated production at $e \bar{e}$ colliders, Phys. Rev. D 52, 3919 (1995).

[7] A. Djouadi, V. Driesen, W. Hollik, and J. Rosiek, Associated production of Higgs bosons and a photon in highenergy $e^{+} e^{-}$collisions, Nucl. Phys. B491, 68 (1997).

[8] A. Barroso, J. Pulido, and J. C. Romao, Higgs production at $e^{+} e^{-}$colliders, Nucl. Phys. B267, 509 (1986).

[9] W.-L. Sang, W. Chen, F. Feng, Y. Jia, and Q.-F. Sun, Nextto-leading-order QCD corrections to $e^{+} e^{-} \rightarrow H+\gamma$, Phys. Lett. B 775, 152 (2017).

[10] G. J. Gounaris, F. M. Renard, and N. D. Vlachos, Tests of anomalous Higgs boson couplings through $e^{-} e^{+} \rightarrow \mathrm{HZ}$ and $H \gamma$, Nucl. Phys. B459, 51 (1996).

[11] U. Mahanta, Effect of TeV scale new physics on the rare process $e^{+} e^{-} \rightarrow H \gamma$, Phys. Lett. B 423, 126 (1998).

[12] H.-Y. Ren, New physics searches with Higgs-photon associated production at the Higgs factory, Chin. Phys. C 39, 113101 (2015).

[13] Q.-H. Cao, H.-R. Wang, and Y. Zhang, Probing $H Z \gamma$ and $H \gamma \gamma$ anomalous couplings in the process $e^{+} e^{-} \rightarrow H \gamma$, Chin. Phys. C 39, 113102 (2015).

[14] G. Li, H.-R. Wang, and S.-h. Zhu, Probing CP-violating $h \bar{t} t$ coupling in $e^{+} e^{-} \rightarrow h \gamma$, Phys. Rev. D 93, 055038 (2016).

[15] A. Kobakhidze, N. Liu, L. Wu, and J. Yue, Implications of $C P$-violating Top-Higgs couplings at LHC and Higgs factories, Phys. Rev. D 95, 015016 (2017).

[16] S. L. Hu, N. Liu, J. Ren, and L. Wu, Revisiting associated production of $125 \mathrm{GeV}$ Higgs boson with a photon at a Higgs factory, J. Phys. G 41, 125004 (2014).

[17] G. J. Gounaris and F. M. Renard, Specific supersimple properties of $e^{-} e^{+} \rightarrow \gamma H$ at high energy, Phys. Rev. D 91, 093002 (2015).

[18] S. Heinemeyer and C. Schappacher, Neutral Higgs boson production at $e^{+} e^{-}$colliders in the complex MSSM: A full one-loop analysis, Eur. Phys. J. C 76, 220 (2016).

[19] A. Arhrib, R. Benbrik, and T.-C. Yuan, Associated production of Higgs at linear collider in the inert Higgs doublet model, Eur. Phys. J. C 74, 2892 (2014).

[20] S. Kanemura, M. Kikuchi, K. Sakurai, and K. Yagyu, H-COUP: A program for one-loop corrected Higgs boson couplings in non-minimal Higgs sectors, Comput. Phys. Commun. 233, 134 (2018).

[21] M. J. Boland et al. (CLICdp and CLIC Collaborations), Updated baseline for a staged compact linear collider, arXiv:1608.07537.

[22] N. G. Deshpande and E. Ma, Pattern of symmetry breaking with two Higgs doublets, Phys. Rev. D 18, 2574 (1978).

[23] R. Barbieri, L. J. Hall, and V. S. Rychkov, Improved naturalness with a heavy Higgs: An alternative road to LHC physics, Phys. Rev. D 74, 015007 (2006).

[24] A. Arhrib, R. Benbrik, J. El Falaki, and A. Jueid, Radiative corrections to the triple Higgs coupling in the inert Higgs doublet model, J. High Energy Phys. 12 (2015) 1.

[25] S. Kanemura, M. Kikuchi, and K. Sakurai, Testing the dark matter scenario in the inert doublet model by future precision measurements of the Higgs boson couplings, Phys. Rev. D 94, 115011 (2016).

[26] B. Swiezewska, Yukawa independent constraints for twoHiggs-doublet models with a $125 \mathrm{GeV}$ Higgs boson, Phys. Rev. D 88, 055027 (2013).

[27] B. Swiezewska and M. Krawczyk, Diphoton rate in the inert doublet model with a $125 \mathrm{GeV}$ Higgs boson, Phys. Rev. D 88, 035019 (2013).

[28] I. F. Ginzburg, K. A. Kanishev, M. Krawczyk, and D. Sokolowska, Evolution of universe to the present inert phase, Phys. Rev. D 82, 123533 (2010).

[29] S. Kanemura, T. Kubota, and E. Takasugi, Lee-QuiggThacker bounds for Higgs boson masses in a two doublet model, Phys. Lett. B 313, 155 (1993).

[30] A. G. Akeroyd, A. Arhrib, and E.-M. Naimi, Note on tree level unitarity in the general two Higgs doublet model, Phys. Lett. B 490, 119 (2000).

[31] B. Grinstein, C. W. Murphy, and P. Uttayarat, One-loop corrections to the perturbative unitarity bounds in the $C P$-conserving two-Higgs doublet model with a softly broken $\mathbb{Z}_{2}$ symmetry, J. High Energy Phys. 06 (2016) 070.

[32] S. Kanemura, T. Kasai, and Y. Okada, Mass bounds of the lightest $C P$ even Higgs boson in the two Higgs doublet model, Phys. Lett. B 471, 182 (1999).

[33] A. Goudelis, B. Herrmann, and O. Stål, Dark matter in the inert doublet model after the discovery of a Higgs-like boson at the LHC, J. High Energy Phys. 09 (2013) 106.

[34] A. Belyaev, G. Cacciapaglia, I. P. Ivanov, F. Rojas-Abatte, and M. Thomas, Anatomy of the inert two Higgs doublet model in the light of the LHC and non-LHC dark matter searches, Phys. Rev. D 97, 035011 (2018).

[35] A. Pierce and J. Thaler, Natural dark matter from an unnatural Higgs boson and new colored particles at the TeV scale, J. High Energy Phys. 08 (2007) 026.

[36] E. Lundstrom, M. Gustafsson, and J. Edsjo, The inert doublet model and LEP II limits, Phys. Rev. D 79, 035013 (2009).

[37] M. Gustafsson, The inert doublet model and its phenomenology, Proc. Sci. CHARGED2010 (2010) 030.

[38] G. Belanger, B. Dumont, A. Goudelis, B. Herrmann, S. Kraml, and D. Sengupta, Dilepton constraints in the inert doublet model from Run 1 of the LHC, Phys. Rev. D 91, 115011 (2015).

[39] E. Ma, Verifiable radiative seesaw mechanism of neutrino mass and dark matter, Phys. Rev. D 73, 077301 (2006).

[40] L. Lopez Honorez, E. Nezri, J. F. Oliver, and M. H. G. Tytgat, The inert doublet model: An Archetype for dark matter, J. Cosmol. Astropart. Phys. 02 (2007) 028.

[41] E. Aprile et al. (XENON Collaboration), First Dark Matter Search Results from the XENON1T Experiment, Phys. Rev. Lett. 119, 181301 (2017).

[42] A. Ilnicka, T. Robens, and T. Stefaniak, Constraining extended scalar sectors at the LHC and beyond, Mod. Phys. Lett. A 33, 1830007 (2018).

[43] T. Hambye, F. S. Ling, L. Lopez Honorez, and J. Rocher, Scalar multiplet dark matter, J. High Energy Phys. 07 (2009) 090.

[44] T. Araki, C. Q. Geng, and K. I. Nagao, Dark matter in inert triplet models, Phys. Rev. D 83, 075014 (2011). 
[45] A. Arhrib, R. Benbrik, M. Chabab, G. Moultaka, M. C. Peyranere, L. Rahili, and J. Ramadan, The Higgs potential in the type II Seesaw model, Phys. Rev. D 84, 095005 (2011).

[46] M. Aoki and S. Kanemura, Unitarity bounds in the Higgs model including triplet fields with custodial symmetry, Phys. Rev. D 77, 095009 (2008).

[47] K. Hartling, K. Kumar, and H. E. Logan, The decoupling limit in the Georgi-Machacek model, Phys. Rev. D 90, 015007 (2014).

[48] E. J. Chun, H. M. Lee, and P. Sharma, Vacuum stability, perturbativity, EWPD and Higgs-to-diphoton rate in type II Seesaw models, J. High Energy Phys. 11 (2012) 106.

[49] G. Abbiendi et al. (OPAL Collaboration), Search for doubly charged Higgs bosons with the OPAL detector at LEP, Phys. Lett. B 526, 221 (2002).

[50] J. Abdallah et al. (DELPHI Collaboration), Search for doubly charged Higgs bosons at LEP-2, Phys. Lett. B 552, 127 (2003).

[51] P. Achard et al. (L3 Collaboration), Search for doubly charged Higgs bosons at LEP, Phys. Lett. B 576, 18 (2003).

[52] V. M. Abazov et al. (D0 Collaboration), Search for Doubly-Charged Higgs Boson Pair Production in $p \bar{p}$ Collisions at $\sqrt{s}=1.96 \mathrm{TeV}$, Phys. Rev. Lett. 108, 021801 (2012).

[53] T. Aaltonen et al. (CDF Collaboration), Search for New Physics in High $p_{T}$ Like-Sign Dilepton Events at CDF II, Phys. Rev. Lett. 107, 181801 (2011).

[54] S. Chatrchyan et al. (CMS Collaboration), A search for a doubly-charged Higgs boson in $p p$ collisions at $\sqrt{s}=7$ TeV, Eur. Phys. J. C 72, 2189 (2012).

[55] G. Aad et al. (ATLAS Collaboration), Search for anomalous production of prompt same-sign lepton pairs and pairproduced doubly charged Higgs bosons with $\sqrt{s}=8 \mathrm{TeV}$ $p p$ collisions using the ATLAS detector, J. High Energy Phys. 03 (2015) 041

[56] S. Yaser Ayazi and S. M. Firouzabadi, Constraining inert triplet dark matter by the LHC and FermiLAT, J. Cosmol. Astropart. Phys. 11 (2014) 005.

[57] A. M. Sirunyan et al. (CMS Collaboration), Observation of Electroweak Production of Same-Sign W Boson Pairs in the Two Jet and Two Same-Sign Lepton Final State in Proton-Proton Collisions at $\sqrt{s}=13 \mathrm{TeV}$, Phys. Rev. Lett. 120, 081801 (2018).

[58] M. Aoki, S. Kanemura, M. Kikuchi, and K. Yagyu, Radiative corrections to the Higgs boson couplings in the triplet model, Phys. Rev. D 87, 015012 (2013).

[59] Y. Kajiyama, H. Okada, and K. Yagyu, Two loop radiative Seesaw model with inert triplet scalar field, Nucl. Phys. B874, 198 (2013).

[60] H. Okada and Y. Orikasa, Radiative neutrino model with an inert triplet scalar, Phys. Rev. D 94, 055002 (2016).

[61] W.-B. Lu and P.-H. Gu, Mixed inert scalar triplet dark matter, radiative neutrino masses and Leptogenesis, Nucl. Phys. B924, 279 (2017).

[62] S. L. Glashow and S. Weinberg, Natural conservation laws for neutral currents, Phys. Rev. D 15, 1958 (1977).
[63] V. D. Barger, J. L. Hewett, and R. J. N. Phillips, New constraints on the charged Higgs sector in two Higgs doublet models, Phys. Rev. D 41, 3421 (1990).

[64] Y. Grossman, Phenomenology of models with more than two Higgs doublets, Nucl. Phys. B426, 355 (1994).

[65] A. G. Akeroyd and W. J. Stirling, Light charged Higgs scalars at high-energy $e^{+} e^{-}$colliders, Nucl. Phys. B447, 3 (1995).

[66] A. G. Akeroyd, Nonminimal neutral Higgs bosons at LEP-2, Phys. Lett. B 377, 95 (1996).

[67] A. G. Akeroyd, Fermiophobic and other nonminimal neutral Higgs bosons at the LHC, J. Phys. G 24, 1983 (1998).

[68] M. Aoki, S. Kanemura, K. Tsumura, and K. Yagyu, Models of Yukawa interaction in the two Higgs doublet model, and their collider phenomenology, Phys. Rev. D 80, 015017 (2009).

[69] M. Sher, Electroweak Higgs potentials and vacuum stability, Phys. Rep. 179, 273 (1989).

[70] I. F. Ginzburg and I. P. Ivanov, Tree-level unitarity constraints in the most general 2HDM, Phys. Rev. D 72, 115010 (2005).

[71] S. Kanemura and K. Yagyu, Unitarity bound in the most general two Higgs doublet model, Phys. Lett. B 751, 289 (2015).

[72] D. Toussaint, Renormalization effects from superheavy Higgs particles, Phys. Rev. D 18, 1626 (1978).

[73] S. Bertolini, Quantum effects in a two Higgs doublet model of the Electroweak interactions, Nucl. Phys. B272, 77 (1986).

[74] M. E. Peskin and J. D. Wells, How can a heavy Higgs boson be consistent with the precision electroweak measurements?, Phys. Rev. D 64, 093003 (2001).

[75] W. Grimus, L. Lavoura, O. M. Ogreid, and P. Osland, The Oblique parameters in multi-Higgs-doublet models, Nucl. Phys. B801, 81 (2008).

[76] S. Kanemura, Y. Okada, H. Taniguchi, and K. Tsumura, Indirect bounds on heavy scalar masses of the two-Higgsdoublet model in light of recent Higgs boson searches, Phys. Lett. B 704, 303 (2011).

[77] A. Arbey, F. Mahmoudi, O. Stal, and T. Stefaniak, Status of the charged Higgs boson in two Higgs doublet models, Eur. Phys. J. C 78, 182 (2018).

[78] M. Misiak and M. Steinhauser, Weak radiative decays of the B meson and bounds on $M_{H^{ \pm}}$in the two-HiggsDoublet model, Eur. Phys. J. C 77, 201 (2017).

[79] S. Kanemura, Y. Okada, E. Senaha, and C. P. Yuan, Higgs coupling constants as a probe of new physics, Phys. Rev. D 70, 115002 (2004).

[80] S. Kanemura, M. Kikuchi, and K. Yagyu, Radiative corrections to the Yukawa coupling constants in two Higgs doublet models, Phys. Lett. B 731, 27 (2014).

[81] S. Kanemura, M. Kikuchi, and K. Yagyu, Fingerprinting the extended Higgs sector using one-loop corrected Higgs boson couplings and future precision measurements, Nucl. Phys. B896, 80 (2015).

[82] S. Kanemura, M. Kikuchi, and K. Yagyu, Radiative corrections to the Higgs boson couplings in the model with an additional real singlet scalar field, Nucl. Phys. B907, 286 (2016). 
[83] S. Kanemura, M. Kikuchi, and K. Yagyu, One-loop corrections to the Higgs self-couplings in the singlet extension, Nucl. Phys. B917, 154 (2017).

[84] S. Kanemura, M. Kikuchi, K. Sakurai, and K. Yagyu, Gauge invariant one-loop corrections to Higgs boson couplings in non-minimal Higgs models, Phys. Rev. D 96, 035014 (2017).

[85] S. Kanemura, M. Kikuchi, K. Mawatari, K. Sakurai, and K. Yagyu, Loop effects on the Higgs decay widths in extended Higgs models, Phys. Lett. B 783, 140 (2018).

[86] Q.-H. Cao, E. Ma, and G. Rajasekaran, Observing the dark scalar doublet and its impact on the standard-model Higgs boson at colliders, Phys. Rev. D 76, 095011 (2007).

[87] A. Arhrib, R. Benbrik, and N. Gaur, $H \rightarrow \gamma \gamma$ in inert Higgs doublet model, Phys. Rev. D 85, 095021 (2012).

[88] M. Krawczyk, D. Sokolowska, P. Swaczyna, and B. Swiezewska, Constraining inert dark matter by $R_{\gamma \gamma}$ and WMAP data, J. High Energy Phys. 09 (2013) 055.

[89] G. Aad et al. (ATLAS and CMS Collaborations), Measurements of the Higgs boson production and decay rates and constraints on its couplings from a combined ATLAS and CMS analysis of the LHC pp collision data at $\sqrt{s}=7$ and $8 \mathrm{TeV}$, J. High Energy Phys. 08 (2016) 045.

[90] A. Alves, E. Ramirez Barreto, A. G. Dias, C. A. de S. Pires, F. S. Queiroz, and P. S. Rodrigues da Silva, Probing 3-3-1 models in Diphoton Higgs boson decay, Phys. Rev. D 84, 115004 (2011).

[91] A. Arhrib, R. Benbrik, M. Chabab, G. Moultaka, and L. Rahili, Higgs boson decay into 2 photons in the type II Seesaw model, J. High Energy Phys. 04 (2012) 136.

[92] S. Kanemura and K. Yagyu, Radiative corrections to electroweak parameters in the Higgs triplet model and implication with the recent Higgs boson searches, Phys. Rev. D 85, 115009 (2012).

[93] A. G. Akeroyd and S. Moretti, Enhancement of $H \rightarrow \gamma \gamma$ from doubly charged scalars in the Higgs triplet model, Phys. Rev. D 86, 035015 (2012).

[94] C.-W. Chiang and K. Yagyu, Higgs boson decays to $\gamma \gamma$ and $Z \gamma$ in models with Higgs extensions, Phys. Rev. D 87, 033003 (2013).

[95] P. S. Bhupal Dev, D. K. Ghosh, N. Okada, and I. Saha, $125 \mathrm{GeV}$ Higgs boson and the type-II Seesaw model, J. High Energy Phys. 03 (2013) 150.

[96] A. Dey, J. Lahiri, and B. Mukhopadhyaya, Extended scalar sectors, effective operators and observed data, J. High Energy Phys. 11 (2018) 127.

[97] G. Aad et al. (ATLAS Collaboration), Constraints on new phenomena via Higgs boson couplings and invisible decays with the ATLAS detector, J. High Energy Phys. 11 (2015) 206.

[98] CMS Collaboration, Summary results of high mass BSM Higgs searches using CMS run-I data, CMS collaboration Report No. CMS-PAS-HIG-16-007.

[99] M. Aaboud et al. (ATLAS Collaboration), Searches for the $Z \gamma$ decay mode of the Higgs boson and for new highmass resonances in $p p$ collisions at $\sqrt{s}=13 \mathrm{TeV}$ with the ATLAS detector, J. High Energy Phys. 10 (2017) 112.

[100] A. M. Sirunyan et al. (CMS Collaboration), Search for the decay of a Higgs boson in the $\ell \ell \gamma$ channel in protonproton collisions at $\sqrt{s}=13 \mathrm{TeV}$, J. High Energy Phys. 11 (2018) 152.

[101] G. Passarino and M. J. G. Veltman, One loop corrections for $e^{+} e^{-}$annihilation into $\mu^{+} \mu^{-}$in the Weinberg model, Nucl. Phys. B160, 151 (1979). 